\title{
VLBI for Gravity Probe B. V. Proper Motion and Parallax of the Guide StarR, IM Pegasi
}

\section{Citation}

Ratner, M. I., N. Bartel, M. F. Bietenholz, D. E. Lebach, J.-F. Lestrade, R. R. Ransom, and I. I. Shapiro. 2012. "VLBI for Gravity Probe B. V. Proper Motion and Parallax of the Guide StarR, IM Pegasi." The Astrophysical Journal Supplement Series 201, no. 1: 1-16.

\section{Published Version}

doi:10.1088/0067-0049/201/1/5

\section{Permanent link}

http://nrs.harvard.edu/urn-3:HUL.InstRepos:11858831

\section{Terms of Use}

This article was downloaded from Harvard University's DASH repository, and is made available under the terms and conditions applicable to Other Posted Material, as set forth at http:// nrs.harvard.edu/urn-3:HUL.InstRepos:dash.current.terms-of-use\#LAA

\section{Share Your Story}

The Harvard community has made this article openly available.

Please share how this access benefits you. Submit a story.

\section{Accessibility}




\title{
VLBI FOR GRAVITY PROBE B. V. PROPER MOTION AND PARALLAX OF THE GUIDE STAR, IM PEGASI
}

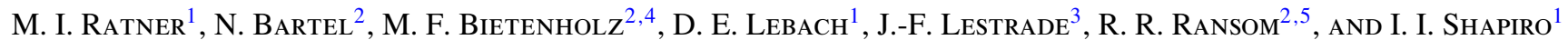 \\ ${ }^{1}$ Harvard-Smithsonian Center for Astrophysics, 60 Garden Street, Cambridge, MA 02138, USA \\ ${ }^{2}$ Department of Physics and Astronomy, York University, 4700 Keele Street, Toronto, ON M3J 1P3, Canada \\ ${ }^{3}$ Observatoire de Paris, Centre National de la Recherche Scientifique, 77 Av. Denfert Rochereau, 75014 Paris, France \\ Received 2011 May 31; accepted 2012 February 23; published 2012 June 7
}

\begin{abstract}
We present the principal astrometric results of the very long baseline interferometry (VLBI) program undertaken in support of the Gravity Probe B (GP-B) relativity mission. VLBI observations of the GP-B guide star, the RS CVn binary IM Pegasi (HR 8703), yielded positions at 35 epochs between 1997 and 2005. We discuss the statistical assumptions behind these results and our methods for estimating the systematic errors. We find the proper motion of IM Peg in an extragalactic reference frame closely related to the International Celestial Reference Frame 2 (ICRF2)

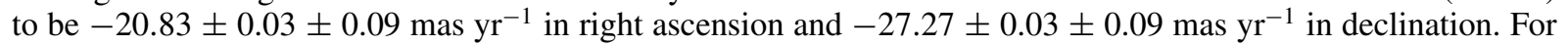
each component, the first uncertainty is the statistical standard error and the second is the total standard error (SE) including plausible systematic errors. We also obtain a parallax of $10.37 \pm 0.07$ mas (distance: $96.4 \pm 0.7 \mathrm{pc}$ ), for which there is no evidence of any significant contribution of systematic error. Our parameter estimates for the $\sim 25$ day period orbital motion of the stellar radio emission have SEs corresponding to $\sim 0.10$ mas on the sky in each coordinate. The total SE of our estimate of IM Peg's proper motion is $\sim 30 \%$ smaller than the accuracy goal

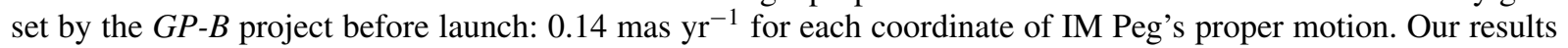
ensure that the uncertainty in IM Peg's proper motion makes only a very small contribution to the uncertainty of the $G P-B$ relativity tests.
\end{abstract}

Key words: astrometry - binaries: close - gravitation - radio continuum: stars - stars: activity - stars: individual (IM Pegasi)

\section{INTRODUCTION}

This paper is the fifth in a series describing the astronomical effort undertaken in support of the NASA/Stanford Gravity Probe B $(G P-B)$ relativity mission, an Earth-orbiting mission to test the geodetic and frame-dragging predictions of general relativity. As described in Paper I (Shapiro et al. 2012), the rotating $G P-B$ spacecraft monitored the precessions of four ultrahigh accuracy onboard gyroscopes with respect to the spacecraft. To transform these precessions to a reference frame that is not rotating with respect to the distant universe, the mission team required both the star-tracking data recorded by the spacecraft's telescope and independent knowledge of the proper motion of an adequately bright "guide" star. Before the launch of $G P-B$, the team set the accuracy requirement

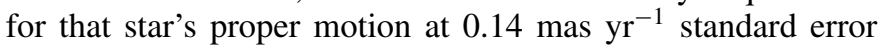
(SE) in each coordinate. Since the proper motion of no bright star was known with such accuracy, we undertook a dedicated program of astrometry to determine this proper motion for the chosen guide star, IM Pegasi (HR 8703). This star is an RS Canum Venaticorum (RS CVn) spectroscopic binary star, with an orbital period of $\sim 25$ days and variable radio emission at centimeter wavelengths. The basic properties of IM Peg, and the requirements and process that led to its selection, are also described in Paper I. To achieve the required astrometric accuracy, we observed IM Peg using the radio astronomical technique of very long baseline interferometry (VLBI) at 35 epochs over a span of 8.5 years.

\footnotetext{
4 Now also at Hartebeesthoek Radio Astronomy Observatory, PO Box 443, Krugersdorp 1740, South Africa.

5 Now at Okanagan College, 583 Duncan Avenue West, Penticton, BC, V2A 2K8, Canada and also at the National Research Council of Canada, Herzberg Institute of Astrophysics, Dominion Radio Astrophysical Observatory, PO Box 248, Penticton, BC, V2A 6K3, Canada.
}

In Section 2, we briefly describe our VLBI observations, while referencing, as appropriate, the earlier papers of this series. Next, in Section 3, we outline the process by which we estimate, for each session of observations, a single effective position for the stellar radio emission. We also comment on the most important sources of error in this process. In Section 4 we describe how we estimate the astrometric parameters of IM Peg from this set of radio positions, then present the resulting parameter estimates and postfit position residuals, and discuss the goodness-of-fit of our model and our estimates of the statistical and systematic errors. We discuss our final results in Section 5, and compare them with those from the Hipparcos mission as well as from ground-based optical observations in Section 6. In Section 7, we summarize our conclusions. Throughout, we use the words "images" and "maps" almost interchangeably.

\section{OBSERVATIONS}

We designed our VLBI program to meet the requirements of the $G P-B$ mission. One important consideration was that we could not rule out the possibility that IM Peg is part of a larger triple or multiple system, and therefore would have a timedependent apparent proper motion. Consequently, we decided to make enough VLBI observations, especially during the years immediately before and after the launch of $G P-B$, to ensure that the proper-motion requirement could be met were there a nearly constant rate of "proper acceleration" (see Section 4.1). Moreover, from the same set of observations we needed to determine IM Peg's parallax and the orbital motion of the radio emission, expected to be associated mainly with only one of the stellar components of the spectroscopic binary. From the time of the selection of IM Peg as the guide star in 1997 (see Paper I), we scheduled, made, and analyzed about four sessions of VLBI 
observations every year until the $G P-B$ mission ended. We thus conducted 35 sessions of VLBI observations of IM Peg between 1997 January and 2005 July.

In each session, observations of IM Peg were interleaved (every 5.5-7.3 minutes) in a repeated cycle with observations of two or three extragalactic reference sources (see below). For most sessions we achieved excellent $u-v$ coverage, good angular resolution, and high sensitivity through the use of full tracks with all available Very Long Baseline Array stations, the Very Large Array, the Effelsberg $100 \mathrm{~m}$ antenna, and the three $70 \mathrm{~m}$ antennas of NASA's Deep Space Network (DSN). Our maximum projected baseline length for most sessions was $\sim 8900 \mathrm{~km}$. During our first two sessions we recorded only rightcircular polarization, with a bit rate of $112 \mathrm{Mbits} \mathrm{s}^{-1}$. During the remaining sessions we recorded both circular polarizations, with a total bit rate of $128 \mathrm{Mbits} \mathrm{s}^{-1}$ in all but the last three of our 35 sessions, for which a $256 \mathrm{Mbits} \mathrm{s}^{-1}$ rate was used. For a full description of our array, typical scan lengths, and data recording parameters, see Paper II (Ransom et al. 2012a). The high sensitivity of this array, together with our use of the technique of phase-referenced mapping, allowed us to map IM Peg even when its flux density fell to as low as $0.2 \mathrm{mJy}$. All the results analyzed below were obtained from observations at $\lambda=3.6 \mathrm{~cm}$, for which the synthesized beam size was typically $\sim 1$ mas $\times \sim 2$ mas. During all but a few sessions, we used only this band, to maximize our detection sensitivity to the unpredictable and highly variable emission from IM Peg, while still cycling rapidly among the sources so as to facilitate phase referencing and reduce many sources of astrometric error. Our strongest and closest reference source, the 7-10 Jy radio-bright quasar 3C 454.3, lying 0.7 mostly south and somewhat east of IM Peg, was observed in all of our sessions, as was the 0.35-0.45 Jy quasar B2250+194 (ICRF J225307.3+194234), which lies 2.9 north of the star. During the final 12 sessions, we also included in our observing cycle the $0.017 \mathrm{Jy}$ compact source B2252+172 (87GB 225231.0+171747), 0.8 northeast of IM Peg, to provide an additional check on the stationarity of our other two reference sources. We added this third reference source after we had gained confidence in the robustness of our data processing procedures and learned that variations in the observed source structure of the other reference sources could contribute significantly to our astrometric uncertainty. As argued in Paper III (Bartel et al. 2012), we assume that this third reference source is also extragalactic, even though its $R=24$ optical counterpart is so faint that no spectrum has yet been obtained for it.

The cadence of our observation sessions was determined by a combination of factors. To ensure that our estimate of proper motion would be only minimally degraded by the need to also estimate a parallax from the same set of astrometric data, we spread the sessions widely across the seasons. Similarly, to allow us to estimate the orbital contribution to the motion of the stellar radio emission, we took care that the sessions were also well distributed in phase with respect to the known binary orbital period of IM Peg. Although the accuracy of the propermotion estimate might have been improved by concentrating the sessions at the beginning and the end of the program, this strategy was effectively precluded by practical considerations regarding the scheduling and analysis of the observations, not to mention the indeterminacy in 1997 of the year of launch (2004) of the $G P-B$ spacecraft, which was several years later than the date intended in 1997. During the last year of the program, on the other hand, the end of the spacecraft operations could be predicted to within an uncertainty of a very few months. Our VLBI observations that year were scheduled on dates which we calculated would lead, once all the anticipated VLBI position measurements were made and analyzed, to relatively high accuracy astrometric parameter estimates and low correlations among those estimates.

In addition to the positions from the 35 sessions scheduled in support of $G P-B$, we had four reliable positions obtained between 1991 and 1994 at the same wavelength by one of us (J.-F.L.) in support of the Hipparcos mission (Lestrade et al. 1995). As described in Paper I, the existence of these VLBI data also played a role in the selection of IM Peg as the $G P-B$ guide star. These earlier observations differed from the others in several ways. In each of the earlier sessions, only four VLBI stations, all on the United States mainland, were used, and the observations of IM Peg were interleaved with observations of only 3C 454.3. The lower sensitivity of the array used for these sessions likely explains why four other similar sessions between 1992 and 1994 yielded either no detection of IM Peg, or, in one case, a relatively weak detection for which no reliable position could be derived. Although the four successful sessions greatly extend our time base, we use the resulting positions with caution, since they might be affected by different measurement errors and possibly a different measurement bias than are the later observations. These four observations are, however, very valuable in addressing the issue of a possible third component in the IM Peg system.

\section{POSITION DETERMINATIONS}

\subsection{Definition of the Stellar Radio Position}

As described in Paper IV (Lebach et al. 2012), we estimate the position of the radio emission of IM Peg at each epoch by a nonstandard, multistep process that includes both phasereferenced mapping and phase-delay fitting with a Kalman-filter estimator. The last major computational task in this process is to produce a phase-referenced map of IM Peg based on the final phase calibrations computed with the Kalman-filter estimator (see Paper IV). These maps are $256 \times 256$ pixels, with a pixel size of 0.15 mas. By construction, we know with acceptable error the coordinate offset of each pixel of this image from the position of our chosen quasi-inertial point in 3C 454.3. Nevertheless, it is not self-evident which position in each image of IM Peg should be used for our subsequent task of estimating IM Peg's proper motion from the full set of positions, because the stellar radio emission is always detectably extended on the sky. Our phase-referenced images reveal this radio emission to fall into three general categories for the radio source structure of IM Peg: (1) single-peaked with peak located near the center of a marginally extended source, (2) single-peaked with peak located noticeably off center from an elongated source, and (3) double-peaked (or in one case apparently triple-peaked) with maximum separation between peaks of $\sim 1.5$ mas. We show an example from each of these categories in Figure 1. See Paper VII (Bietenholz et al. 2012) for the complete set of images and a discussion of them. Given that the stellar radio emission was detectably extended, we attempted to find some feature or pattern in our images with an unambiguous spatial relationship to any other physically meaningful location in the rotating binary system. Unfortunately, we were unable to find any such unambiguous relationship. We therefore decided to fit an orbit to the "center" of the radio emission defined as 

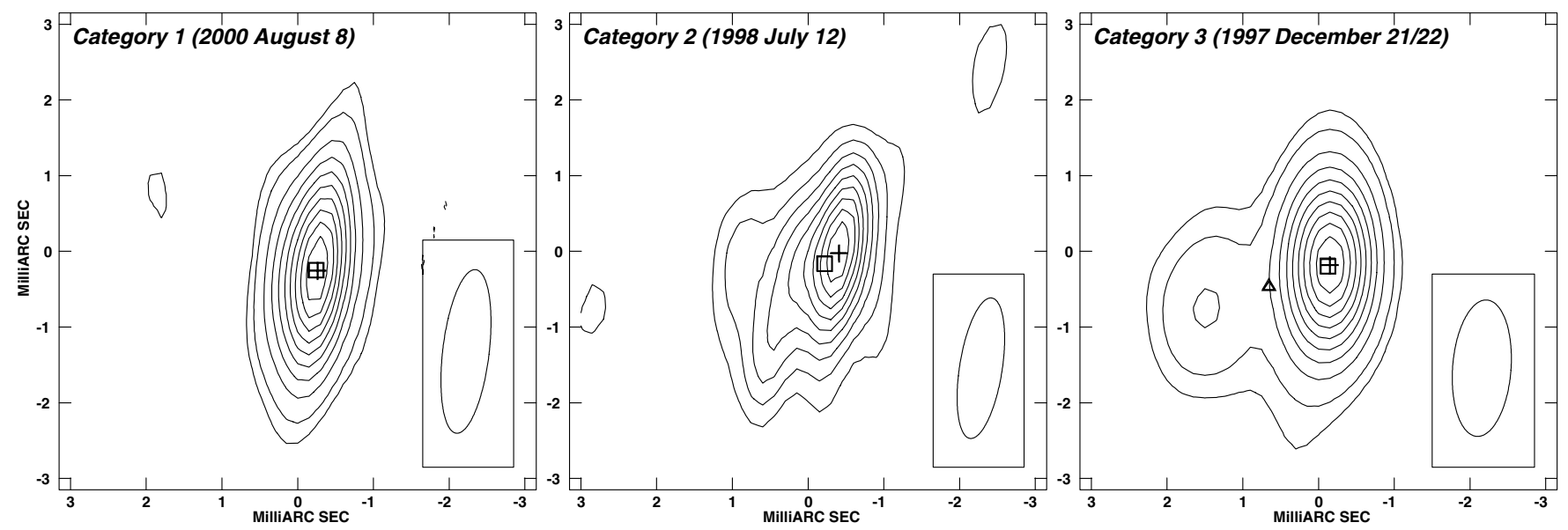

Figure 1. Selection of (Stokes $I, 8.4 \mathrm{GHz}$ ) images of IM Peg illustrating the three categories of source structures (see Section 3.1). The epoch to which each image corresponds is given in parentheses. The full set of images of IM Peg is presented in Paper VII. The brightness peak in each image is $32.7 \mathrm{mJy}^{-1}$ beam ${ }^{-1}$ (2000 August 8), $0.72 \mathrm{mJy} \mathrm{beam}^{-1}$ (1998 July 12), and $31.9 \mathrm{mJy} \mathrm{beam}^{-1}$ (1997 December 21/22). The contour levels displayed in the images for 2000 August 8 and 1997 December $21 / 22$ are $-5 \%$ (dotted), $5 \%, 10 \%, 20 \%, 30 \%, 40 \%, 50 \%, 60 \%, 70 \%, 80 \%$, and $90 \%$ of the peak brightness for that image. The contour levels displayed in the image for 1998 July 12 are the same, except that the lowest contour is $10 \%$. The restoring beam is shown in the bottom right-hand corner of each image. North is up and east is to the left. The small cross in each image represents the position of the brightness peak. The small open square in each image represents the position of the peak of a single elliptical Gaussian fit to a region about the source (and down to the noise level of the image). The small open triangle in the image for 1997 December $21 / 22$ is the position of the midpoint between the eastern and western peaks.

follows: for each of the sessions for which the radio image of IM Peg contained only one, centered, clearly detected local maximum (Category 1, Figure 1), we took the center of the two-dimensional Gaussian component obtained by fitting such a component (using AIPS task IMFIT or JMFIT) to the brightest part of the image. For Category 2 epochs, we considered two choices. The first is to interpolate the brightness-peak position directly from the image, performing a quadratic interpolation between the pixel brightnesses (via MAXFIT in AIPS) to obtain sub-pixel accuracy on the position of the brightness peak. The second is to use the position of the peak of a Gaussian component fit to the image (see Figure 1). For Category 3 epochs, we considered three choices, namely, the overall brightness peak, the peak of a single Gaussian component fit to the entire region of detected emission, and the midpoint between or among the two or three local brightness maxima (see Figure 1). (For Category 1 epochs, the difference between the position of the interpolated brightness peak and the peak of the fit Gaussian component is in all cases $<0.07$ mas, and in most cases $<0.03$ mas, and hence insignificant.) We initially reserved judgment as to the "best" choice, and prepared three sets of positions for our 35 epochs: (1) the position of the interpolated brightness peak for each epoch, (2) the position of the center of the single Gaussian for each epoch, and (3) the position of the center of the single Gaussian for each single-peaked epoch and the positions of the unweighted midpoint for each multiple-peaked epoch. We then fit the astrometric model described below in Section 4.1 to each of the three sets of positions. We obtained the best fit for choice (3): the chi-square per degree of freedom for the resulting fit was $20 \%$ lower than that obtained for choice (2) and 30\% lower than that for choice (1). We therefore adopted set (3) as our standard set of astrometric positions for IM Peg.

For each of our VLBI sessions we also had to specify the sky coordinates for some reference point in our image of $3 \mathrm{C} 454.3$. For all sessions from 1997 onward, we chose this point to be the peak of the $\mathrm{C} 1$ component near the core of 3C 454.3. In Paper III, we find that $\mathrm{C} 1$ is stationary in a nearly inertial, extragalactic, reference frame. For the four earlier epochs, for which our maps have insufficient resolution to adequately resolve this component, we used the brightness peak of the core as the reference point, and assumed that its coordinates are offset from those of $\mathrm{C} 1$ by the average amount of that offset determined for the later epochs $(-0.26$ mas in $\alpha$ and -0.03 mas in $\delta)$.

\subsection{Estimated Positions and Their Errors}

The IM Peg positions resulting from this process are given in Table 1. The uncertainties of these IM Peg positions are dominated by poorly characterized systematic errors, whose size we can estimate from the scatter in the differential positions we found for our pair of reference sources with the smallest separation on the sky, B2252+172 and 3C 454.3. Since the angular separation of even this pair is about twice that of IM Peg from 3C 454.3, this approach should yield conservative uncertainties. For the sessions from 1997 through 2005, we estimate our uncertainty based on the weighted root-meansquare (weighted rms) scatter, 0.045 mas in $\alpha$ and 0.037 mas in $\delta$, of the postfit residuals that we obtained for the 12 (2002-2005) positions of B2252+172 with respect to 3C 454.3 (see Paper III). However, in light of the noticeably larger scatter seen in the B2250+194 positions in the years before B2252+172 was observed, we multiply the rms scatter in the B2252+172 positions by the ratio of the rms residual scatter for each coordinate of B2250+194 (with respect to 3C 454.3) for the entire period 1997-2005 to the rms residual scatter found for only the 12 sessions during which B2252+172 was observed. The result is an uncertainty of $\sim 0.06$ mas in each coordinate. In addition, there is a nearly constant, common error in all these positions, due to the uncertainty of the position of C1. In Paper III we show that, from 1998 through our last VLBI observation in 2005, its mean (J2000) coordinates are $22^{\mathrm{h}} 53^{\mathrm{m}} 57^{\mathrm{s}} .7479573(31) 16^{\circ} 08^{\prime} 53^{\prime \prime} .561281$ (68), where the SEs for the last two digits are given in parentheses. Over the same span, this component was stationary in our extragalactic reference frame to within our estimated $1 \sigma$ bounds of 0.046 and 0.056 mas $\mathrm{yr}^{-1}$, in $\alpha$ and $\delta$, respectively. We cannot make a similar inference of the measurement uncertainties in the four IM Peg positions we derived from the 1991-1994 VLBI sessions. Given the smaller VLBI array used during these sessions and the resulting poorer angular resolution and 
Table 1

IM Peg Position Estimates ${ }^{\mathrm{a}}$

\begin{tabular}{|c|c|c|c|c|}
\hline \multirow{2}{*}{$\begin{array}{l}\text { Observation } \\
\text { Date }\end{array}$} & \multirow{2}{*}{$\begin{array}{l}\text { Epoch } \\
\text { MJDb }^{\mathrm{b}}\end{array}$} & \multicolumn{2}{|c|}{ Position } & \multirow{2}{*}{$\begin{array}{l}\text { No. of Image } \\
\text { Components }\end{array}$} \\
\hline & & $\begin{array}{c}\alpha-22^{\mathrm{h}} 53^{\mathrm{m}} 2^{\mathrm{s}} .0 \\
(\mathrm{~s})\end{array}$ & $\begin{array}{c}\delta-16^{\circ} 50^{\prime} 28^{\prime \prime} .0 \\
\left({ }^{\prime \prime}\right)\end{array}$ & \\
\hline 19911215 & 48605.06 & 0.276990 & 0.51462 & 1 \\
\hline 19930622 & 49160.51 & 0.276091 & 0.48065 & 1 \\
\hline 19930913 & 49243.25 & 0.274992 & 0.47385 & 1 \\
\hline 19940723 & 49556.45 & 0.274324 & 0.45323 & 1 \\
\hline 19970116 & 50464.90 & 0.269798 & 0.37361 & 2 \\
\hline 19970118 & 50466.89 & 0.269812 & 0.37288 & 1 \\
\hline 19971130 & 50782.03 & 0.268379 & 0.35337 & 1 \\
\hline 19971221 & 50803.96 & 0.268350 & 0.34949 & 2 \\
\hline 19971227 & 50809.96 & 0.268352 & 0.34835 & 2 \\
\hline 19980301 & 50873.78 & 0.268689 & 0.34413 & 1 \\
\hline 19980712 & 51006.41 & 0.268662 & 0.34443 & 1 \\
\hline 19980808 & 51033.35 & 0.268368 & 0.34217 & 1 \\
\hline 19980917 & 51073.24 & 0.267747 & 0.33847 & 1 \\
\hline 19990313 & 51250.74 & 0.267332 & 0.31730 & 1 \\
\hline 19990515 & 51313.57 & 0.267509 & 0.31785 & 2 \\
\hline 19990919 & 51440.23 & 0.266273 & 0.30996 & 2 \\
\hline 19991209 & 51521.99 & 0.265512 & 0.29712 & 2 \\
\hline 20000515 & 51679.56 & 0.266060 & 0.29084 & 1 \\
\hline 20000807 & 51763.34 & 0.265419 & 0.28893 & 1 \\
\hline 20001106 & 51854.09 & 0.264135 & 0.27355 & 1 \\
\hline 20001107 & 51855.01 & 0.264148 & 0.27339 & 1 \\
\hline 20010331 & 51999.73 & 0.264494 & 0.26210 & 1 \\
\hline 20010629 & 52089.48 & 0.264483 & 0.26372 & 1 \\
\hline 20011020 & 52202.05 & 0.262867 & 0.25081 & 1 \\
\hline 20011221 & 52264.99 & 0.262528 & 0.24069 & 1 \\
\hline 20020414 & 52378.65 & 0.263190 & 0.23659 & 1 \\
\hline 20020714 & 52469.40 & 0.262771 & 0.23533 & 1 \\
\hline 20021121 & 52599.06 & 0.261148 & 0.21821 & 1 \\
\hline 20030126 & 52665.88 & 0.261121 & 0.20940 & 1 \\
\hline 20030518 & 52777.55 & 0.261785 & 0.21022 & 2 \\
\hline 20030909 & 52891.24 & 0.260541 & 0.20210 & 1 \\
\hline 20031206 & 52979.00 & 0.259686 & 0.18744 & 3 \\
\hline 20040306 & 53070.76 & 0.260047 & 0.18194 & 2 \\
\hline 20040518 & 53143.58 & 0.260287 & 0.18250 & 1 \\
\hline 20040626 & 53182.49 & 0.260099 & 0.18041 & 1 \\
\hline 20041212 & 53351.00 & 0.258124 & 0.15938 & 1 \\
\hline 20050115 & 53385.92 & 0.258220 & 0.15548 & 1 \\
\hline 20050528 & 53518.45 & 0.258850 & 0.15447 & 1 \\
\hline 20050716 & 53567.41 & 0.258498 & 0.15343 & 1 \\
\hline
\end{tabular}

\section{Notes.}

${ }^{\text {a }}$ For our estimates of the uncertainties of these positions, see Section 3.2.

b Modified Julian Date $=$ Julian Date $-2,400,000.5$ days.

$u-v$ coverage, and given that only one reference source (3C 454.3) was observed, the uncertainty for these four positions could plausibly be up to about twice as large as that of the other IM Peg positions. In any case, no estimate of the measurement uncertainty in our IM Peg positions was used in the rest of our astrometric analysis, because, as expected, this uncertainty was for all sessions much smaller than the rms of the seemingly random scatter of our postfit position residuals, which is $\sim 0.4$ mas in each coordinate (see Table 2 and Section 4.2). This scatter is evidently dominated not by the measurement noise, but rather by some other seemingly noiselike contribution.

The largest source of scatter is likely a highly variable spatial offset between the stellar radio emission and the center of the primary component of the IM Peg binary. The strongest evidence for this assertion is that, for some of those VLBI sessions marked by emission strong enough to be detectable in single scans, our VLBI astrometry reveals changes in position of up to $\sim 1$ mas occurring in synchrony with changes in the brightness of the emission (Lebach et al. 1999). In addition, it is plausible that the radio emission is both powered and loosely confined by the stellar magnetic field (Franciosini \& Chiuderi Drago 1995). Moreover, the photospheric spot maps derived from optical spectroscopy (e.g., Berdyugina et al. 2000) imply that the stellar magnetic field is highly variable and asymmetric. It is therefore not surprising that the peak of the radio emission is displaced from the center of the star in a seemingly random manner by an amount comparable to the $\sim 0.6$ mas angular radius of the primary. For further discussion, see Section 4.3 below and Papers VI (Ransom et al. 2012b) and VII.

Consequently, we assume that the size and possible anisotropy of the uncertainty of the VLBI-derived IM Peg positions are best determined from the positions themselves. Since we find no convincing evidence for any systematic variation in that uncertainty, we assign identical uncertainty to all of our IM Peg positions, including those for the pre-1997 sessions.

Smaller position errors are contributed by (1) errors in identifying the reference point in our maps of 3C 454.3 and (2) inaccuracies in our astrometric model (including the various inputs to that model). Some of these errors vary on timescales of months or years and thus cause non-negligible correlations between the errors of our estimated IM Peg positions for sessions that were months or even years apart. In fact, the correlations of such errors are evident in the tendency of the estimated relative positions of our reference sources to vary slowly with time, rather than exhibit a white-noise behavior (see Paper III). Moreover, these correlations are not unexpected for two reasons. First, it is known that the flux density and also the structure (i.e., shape) of the emitting regions of 3C 454.3 each exhibits strong autocorrelations over many months (see Paper II). Second, the ionospheric total electron content also correlates over several years, as it rises and falls with the $\sim 11$ year sunspot cycle. The resulting position error likely also has a nonzero correlation over several years. Moreover, as discussed in Paper III, the uncertainty in our position estimates caused by inaccuracies in our ionosphere models is one of our major sources of error. Conservative estimated standard deviations (see Paper III for details) on the contributions of errors in our modeling of the propagation medium-ionosphere plus troposphere- to the position differences among our reference sources range up to $\sim 0.1$ mas. Hence, given the smaller angular separation between IM Peg and 3C 454.3, we expect up to $\sim 0.02$ mas for the corresponding error contribution to our IM Peg positions. Any positive correlation among the position measurement errors (or the unmodeled radio position offsets) for epochs separated by up to several years would prevent the uncertainty of our proper-motion estimate from falling in proportion to the square-root of the temporal density of our measured IM Peg positions, as it would for statistically independent position measurements. Slowly varying position errors could thus increase the SE of our proper-motion estimate as much as uncorrelated errors that are severalfold larger. However, the variations in our estimates (in Paper III) of the relative positions of our reference sources are severalfold smaller than the slowly varying component of the postfit residuals of our IM Peg solutions. (For B2252+172 and 3C 454.3 , the closest pair, with separation $\sim 1.4$ on the sky, the weighted rms scatter of the position differences is 0.023 mas in $\alpha$ and 0.051 mas in $\delta$.) The small size of these variations 
Table 2

Comparison of Astrometric Solutions ${ }^{\mathrm{a}}$

\begin{tabular}{|c|c|c|c|c|c|c|c|}
\hline \multirow[t]{2}{*}{ Solution } & \multicolumn{2}{|c|}{ Proper Motion } & \multirow{2}{*}{$\begin{array}{c}\text { Parallax } \\
\pi-10.37 \\
\text { (mas) }\end{array}$} & \multicolumn{2}{|c|}{ Proper Acceleration } & \multicolumn{2}{|c|}{ rms Residuals } \\
\hline & $\begin{array}{c}\mu_{\alpha *}+20.83 \\
\left(\operatorname{mas~yr}^{-1}\right)\end{array}$ & $\begin{array}{l}\mu_{\delta}+27.27 \\
\left(\operatorname{mas~yr}^{-1}\right)\end{array}$ & & $\begin{array}{c}\dot{\mu}_{\alpha *} \\
\left(\operatorname{mas~yr}^{-2}\right)\end{array}$ & $\begin{array}{c}\dot{\mu}_{\delta} \\
\left(\operatorname{mas~yr}^{-2}\right)\end{array}$ & $\begin{array}{c}\alpha \\
(\mathrm{mas})\end{array}$ & $\begin{array}{c}\delta \\
(\mathrm{mas})\end{array}$ \\
\hline 1. Chosen ${ }^{\mathrm{b}}$ & $0.00 \pm 0.03$ & $0.00 \pm 0.03$ & $0.00 \pm 0.07$ & & & 0.354 & 0.416 \\
\hline 2. With proper acceleration ${ }^{b}$ & $-0.07 \pm 0.10$ & $-0.31 \pm 0.10$ & $-0.03 \pm 0.07$ & $-0.018 \pm 0.025$ & $-0.085 \pm 0.025$ & 0.349 & 0.357 \\
\hline 3. With the four early epochs ${ }^{b}$ & $+0.01 \pm 0.02$ & $-0.01 \pm 0.02$ & $+0.01 \pm 0.07$ & $\ldots$ & $\ldots$ & 0.340 & 0.440 \\
\hline 4. With four early epochs and accel. ${ }^{b}$ & $-0.03 \pm 0.05$ & $-0.04 \pm 0.06$ & $+0.01 \pm 0.07$ & $-0.008 \pm 0.008$ & $-0.006 \pm 0.011$ & 0.336 & 0.437 \\
\hline 6. Using "AIPS-only" positions ${ }^{\mathrm{b}}$ & $-0.05 \pm 0.03$ & $+0.05 \pm 0.03$ & $+0.07 \pm 0.08$ & $\ldots$ & $\ldots$ & 0.394 & 0.446 \\
\hline 7. Without last five epochs ${ }^{\mathrm{d}}$ & $0.00 \pm 0.03$ & $+0.07 \pm 0.04$ & $+0.02 \pm 0.08$ & $\ldots$ & $\ldots$ & 0.349 & 0.383 \\
\hline
\end{tabular}

Notes.

${ }^{a}$ Here and elsewhere, the $\alpha$ component of proper motion, $\mu_{\alpha *}$, and its time derivative, $\dot{\mu}_{\alpha *}$, have been multiplied by the factor cos $\delta$, so that they are, respectively, the rates of motion and acceleration on the sky, i.e., $\mu_{\alpha *}=\mu_{\alpha} \cos \delta$, where $\mu_{\alpha}$ is the time derivative of right ascension, $\alpha$. The errors shown are the SSEs yielded for each parameter by the WLS fits. Throughout this paper, we employ J2000 coordinates. For Solutions 2 and 4 , the tabulated proper motion is for epoch JD 2453403.0 (2005 February 1, 2005.08), the approximate midpoint of the GP-B science data.

b See Section 4.2.

c This solution was obtained after excluding the nine epochs for which the stellar radio source exhibited more than one brightness peak (see Table 1).

d See Section 4.3.2.

implies that neither the correlated position error due to reference source structure nor that due to the ionosphere likely accounts for the bulk of the systematic component of our IM Peg position residuals. We postpone a more quantitative treatment of correlated errors to Section 4.3 where we discuss the uncertainty of our results.

\section{ASTROMETRIC SOLUTIONS}

\subsection{The Model}

We use a conventional weighted-least-squares (WLS) technique to fit a linearized model to the IM Peg position estimates described above. The parameters required, in addition to the proper motion of IM Peg, are its position at a reference epoch, its parallax, and four scalar parameters to specify the projection on the sky of its (assumed) zero-eccentricity orbit of accurately known period-nine parameters in all. As discussed below, we considered assigning time-dependent SEs to our VLBI position estimates, and we searched for evidence of nonuniform scatter in our residuals. Nevertheless, since we found no such evidence and had no a priori reason to expect any significant nonuniformity, we used uniform weighting to obtain our final estimates. On the other hand, we found significantly different rms values for the $\alpha$ and $\delta$ components of our postfit residuals, so we allow for such unequal noise levels in the two coordinates and also for a nonzero correlation between their errors. That is, we allow for errors characterized by an error ellipse of arbitrary axial ratio and orientation on the sky. We estimate the required SEs of the $\alpha$ and $\delta$ components of our VLBI positions from the rms value of the postfit residuals in each coordinate, and we estimate the correlation between the two coordinates of the error from the correlation between the same-epoch postfit $\alpha$ and $\delta$ residuals, by the following procedure: to obtain for each coordinate an unbiased estimate of the rms measurement noise value, we increase the rms residual value to account for the number of degrees of freedom taken out of the residuals in the process of estimating our free parameters. Since there is no known bias between the observed same-epoch correlation of the postfit $\alpha$ and $\delta$ residuals and the same-epoch correlation in the $\alpha$ and $\delta$ components of the VLBI position noise, for the latter correlation we adopt the observed correlation without modification.
We found that a single iteration of this procedure, starting with the residuals obtained under the assumption of equal and uncorrelated errors in the $\alpha$ and $\delta$ components, converged to a limiting value (as estimated from an additional iteration) for each estimated parameter, to within $1 \%$ of its statistical standard error (SSE). Thus, for our postfit residuals, $\chi^{2}$ per degree of freedom is unity and, consequently, the SSEs yielded by our WLS fits are unbiased estimates, at least in the approximation that our errors at different epochs are mutually independent and identically distributed.

When we state (above) that our model is a "linearized" rather than "linear" one, we are merely acknowledging that the change in $\alpha$ due to a given angular offset on the sky depends (nonlinearly) on the corresponding $\delta$. However, since $\delta$ is already known to a small fraction of an arcsecond, the partial derivatives of the model with respect to its parameters do not, in practice, have to be recomputed iteratively in our estimation software. Moreover, as described below, we can choose our parameterization of the orbital motion of the radio emission to ensure that the orbital part of our model is linear too.

We use four choices of reference epoch for our positions. Usually, we choose the reference epoch to approximate the effective midpoint of the $G P-B$ flight mission, but to facilitate comparisons with other results (see Section 6) we also computed positions at other epochs, namely the midpoint of our VLBI data, the reference epoch of the Hipparcos Catalogue (ESA 1997), and J2000.0. Regardless of our choice of reference epoch, all of our calculations and results are obtained and presented in J2000 coordinates.

We compute the parallactic offset numerically at each observing epoch using a numerical ephemeris (PEP740R, J. Chandler 1999, private communication) of the heliocentric orbits of the Earth, planets, larger asteroids, and Pluto, and the geocentric lunar orbit, too, though only Jupiter and Saturn actually affect the parallactic offset of IM Peg to a non-negligible degree. The aberration effect of Earth's motion is generally far larger; the largest aberration term, the annual aberration, can be as much as $\sim 20^{\prime \prime}$. However, because the models used in VLBI data processing are always computed in a solar-system barycentric reference frame, all the known aberration effects are removed from the VLBI data at an early stage of processing. At no 
stage of our analysis do the ephemerides contribute more than a (negligible) few microarcseconds of uncertainty to our position results.

We restrict our model to include only a zero-eccentricity orbit, because our data set is far less sensitive to eccentricity than is optical spectroscopy, which bounds any true eccentricity of the IM Peg binary orbit below $~ 0.01$ (Berdyugina et al. 1999; Marsden et al. 2005). Since our 35 VLBI position estimates are characterized by $\sim 0.4$ mas rms noiselike scatter in each coordinate and our WLS orbit of the radio emission on the sky has a semimajor axis of only $\sim 0.9$ mas, the effect of such small eccentricities is not detectable with our data set. Moreover, using software that allows eccentric orbits, developed as part of the Hipparcos frame-tie program of Lestrade et al. (1995), we confirmed that the improvement in the fit to our VLBIdetermined positions achievable with a grid search over all possible values of eccentricity and time of periastron passage is not statistically significant. In any case, the resulting change in our estimate of proper motion would be no more than a

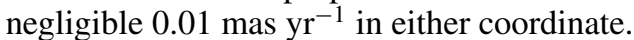

Our VLBI position estimates also lack the precision necessary to detect plausible departures of the orbital period of the radio emission from the value derived from optical data. Even if, to increase the time span of our data set, we include (with equal weight) the four available VLBI positions from 1991 to 1994, the SSE of our WLS estimate of orbital period is $\sim 0.01$ day, which is more than a factor of 300 larger than that of the spectroscopic result of Marsden et al. (2005). Moreover, our WLS adjustment to that result is not statistically significant. Given the lack of any convincing evidence of period variation and the lack of any strong reason to believe that the source of the radio emission drifts systematically with respect to the line connecting the two stellar components of the IM Peg binary, we adopt the optical value for the orbital period (24.64877 days; Marsden et al. 2005). We considered the advisability of also adopting optically derived values for the inclination and the position along the orbit. However, the optically derived inclination has only similar accuracy to ours (see Section 6) and is obtained only indirectly (by combining radial velocities from spectroscopy with mass estimates based on results from spectroscopy and photometry). Furthermore, we cannot rule out the possibility that some type of interaction between the two components of the binary causes the peak of the radio emission, on average, to either lag or precede the position of the primary in its orbit. We therefore estimate these two orbital parameters from our VLBI data, without any a priori constraint on their values.

We usually represent the orbital motion in each coordinate by the sum of one term proportional to the sine of orbital phase and one proportional to the cosine. The resulting model is then strictly linear in the unknown amplitudes. (For the mathematical formulation of these terms, see note " $e$ " to Table 3 .) The choice of the zero point of orbital phase has no effect on the final fit to our VLBI positions or on the values of the nonorbital parameters. To facilitate comparison with the optical results of Marsden et al., we take as our zero point the epoch that they determined to be a (heliocentric) time of conjunction with the primary farther from us than the secondary: Julian day 2,450,342.905. To further aid such comparisons, we can alternatively parameterize the orbital motion by its semimajor axis, axial ratio (as projected on the sky), nodal position angle, and time of conjunction. Since the orbit model is not a linear function of these four alternate parameters, we compute them by iterating their linearized WLS estimates to convergence. As in the previously mentioned case of our iterating to determine the SEs of the $\alpha$ and $\delta$ components of our VLBI positions, convergence of the orbit parameters is reached after just a few iterations. The orbit on the sky specified by our converged WLS estimates of the alternative parameters is identical (to within our fully adequate computational precision) to that obtained with our model parameterized with separate sinusoidal terms in $\alpha$ and $\delta$. Consequently, neither the postfit residuals nor the estimate of any nonorbital parameter differs between the two model parameterizations. Numerical confirmation of this lack of change provided us with a useful check on our WLS fitting software.

Should our model also allow for the possibility that the close binary system is orbited by a third, more distant, companion? One cannot rule out such a companion on astrophysical grounds; on the contrary, about half of known close binaries have more distant stellar companions (e.g., Mayor \& Mazeh 1987; Tokovinin et al. 2006). Moreover, the potential impact of such a companion on the accuracy of our VLBI propermotion estimate is too large to ignore. For example, a star of 1 solar mass separated from the IM Peg binary by $1^{\prime \prime}$ on the sky could cause an angular acceleration of the binary at a rate as high as 0.05 mas $\mathrm{yr}^{-2}$, with the maximum acceleration occurring when the distance of the star from us is identical to that of the binary. If our estimate of proper motion were used in the mission data analysis without taking account of such a possible acceleration, an unacceptably large error in the $G P-B$ relativity tests might be introduced because the mean epoch of our VLBI data is $\sim 4$ yr earlier than that of the spacecraft gyro measurements. We addressed this potential problem in several ways. First, we initiated a campaign of optical observations using the Hubble Space Telescope (HST) and groundbased telescopes to place limits on the maximum brightness of any third companion to the binary (see Section 4.2). Second, we designed our program of VLBI observations to meet the $G P-B$ accuracy requirement for proper motion, even were we to solve for a constant acceleration on the sky-what we call "proper acceleration"-along with the other required parameters. This acceleration term would allow us to remove most of the effect on the $G P-B$ experiment of a companion in an orbit with a period that is too long to be clearly identifiable in our postfit residuals. Later, during our data analysis, we compared the quality of our fits to our VLBI-determined positions with and without adding proper acceleration to our model. As described below in Section 4.3, we concluded that retention of this term is not justified. (Admittedly, an extremely eccentric orbit whose periastron passage occurred during the span of our VLBI data would be modeled poorly in either case, but such orbits and timing are a priori unlikely.) Finally, we visually inspected our postfit residuals and also performed a periodogram analysis of them. We found no clear indication of a periodic component, and hence no need to incorporate a second orbit in our model.

\subsection{Sensitivity of the Results to Various Analysis Options}

In Table 2, we present the proper-motion and parallax estimates from seven different WLS astrometric solutions, which, taken together, justify our reliance upon the first one for our final results. We obtained this Solution 1 by fitting our nineparameter model (without proper acceleration) to the IM Peg positions we determined for all $35 \mathrm{VLBI}$ sessions scheduled in support of $G P-B$. A plot of the fit of this solution to all the position data is shown in Figure 2. The corresponding postfit residuals are plotted in Figure 3. Solution 2 differs from 
Table 3

Final IM Peg Parameter Estimates

\begin{tabular}{|c|c|c|c|c|}
\hline Parameter & Estimate & SSE & Systematic Error ${ }^{\mathrm{a}}$ & Total $\mathrm{SE}^{\mathrm{b}}$ \\
\hline \multicolumn{5}{|l|}{ Nonorbit parameters: } \\
\hline$\alpha$ at epoch $2005.08^{c}$ (errors in mas) & $22^{\mathrm{h}} 53^{\mathrm{m}} 2^{\mathrm{s}} .258612$ & 0.12 & 0.33 & 0.40 \\
\hline$\delta$ at epoch $2005.08^{\mathrm{c}}$ (errors in mas) & $16^{\circ} 50^{\prime} 28^{\prime \prime} .16005$ & 0.13 & 0.29 & 0.39 \\
\hline$\mu_{\alpha *}{ }^{\mathrm{d}}\left(\mathrm{mas} \mathrm{yr}^{-1}\right)$ & -20.833 & 0.026 & 0.073 & 0.090 \\
\hline$\mu_{\delta}\left({\left.\operatorname{mas~} \mathrm{yr}^{-1}\right)}^{-1}\right.$ & -27.267 & 0.030 & 0.074 & 0.095 \\
\hline Parallax (mas) & 10.370 & 0.074 & $<0.015$ & 0.074 \\
\hline \multicolumn{5}{|l|}{ Linear model orbit parameters: ${ }^{\mathrm{e}}$} \\
\hline$A_{\mathrm{s} \alpha}(\mathrm{mas})$ & -0.59 & 0.10 & $\ll 0.1$ & 0.10 \\
\hline$A_{\mathrm{s} \delta}(\mathrm{mas})$ & -0.66 & 0.11 & $\ll 0.1$ & 0.11 \\
\hline$A_{\mathrm{c} \alpha}$ (mas) & 0.15 & 0.09 & $\ll 0.1$ & 0.09 \\
\hline$A_{\mathrm{c} \delta}(\mathrm{mas})$ & -0.23 & 0.11 & $\ll 0.1$ & 0.11 \\
\hline \multicolumn{5}{|l|}{ Alternative orbit parameters: ${ }^{f}$} \\
\hline Semimajor axis (mas) & 0.89 & 0.09 & $\ll 0.1$ & 0.09 \\
\hline Axial ratiog & 0.30 & 0.13 & $\ll 0.1$ & 0.13 \\
\hline$T_{\text {conj }}\left(\right.$ heliocentric JD) ${ }^{\mathrm{i}}$ & 2450342.56 & 0.44 & $\ll 0.4$ & 0.44 \\
\hline \multicolumn{5}{|c|}{ Positions at alternative reference epochs ${ }^{\mathrm{f}}$ (errors in mas): } \\
\hline$\alpha$ at epoch 2001.29 & $22^{\mathrm{h}} 53^{\mathrm{m}} 2^{\mathrm{s}} \cdot 264124$ & 0.07 & 0.30 & 0.35 \\
\hline$\delta$ at epoch 2001.29 & $16^{\circ} 50^{\prime} 28^{\prime \prime} .26362$ & 0.08 & 0.30 & 0.34 \\
\hline$\alpha$ at epoch $\mathrm{J} 2000$ & $22^{\mathrm{h}} 53^{\mathrm{m}} 2^{\mathrm{s}} \cdot 265997$ & 0.07 & 0.35 & 0.38 \\
\hline$\delta$ at epoch $\mathbf{J} 2000$ & $16^{\circ} 50^{\prime} 28^{\prime \prime} .29883$ & 0.09 & 0.36 & 0.40 \\
\hline$\alpha$ at epoch 1991.25 & $22^{\mathrm{h}} 53^{\mathrm{m}} 2^{\mathrm{s}} .278694$ & 0.27 & 0.89 & 1.04 \\
\hline$\delta$ at epoch 1991.25 & $16^{\circ} 50^{\prime} 28^{\prime \prime} .53741$ & 0.31 & 0.94 & 1.13 \\
\hline
\end{tabular}

Notes.

${ }^{a}$ The uncertainties in the position and proper motion of the phase reference point $\mathrm{C} 1$ in $3 \mathrm{C} 454.3$ are included here, and not in the SSE. The uncertainty due to the possible offset and secular drift between the mean position of the stellar radio emission and the center of mass of the binary are likewise included here. However, the upper bounds on the systematic errors in the orbit terms apply to the mean orbit of the radio emission, and not to the corresponding orbital terms for the stellar binary.

b Each "total SE" is our estimate of the parameter's SE, computed as the root-sum-square (RSS) of the SSE and our estimated systematic error. For the position and proper-motion parameters, we first doubled the SSE before computing the RSS, to allow for correlated "noise" in the VLBI positions.

${ }^{\mathrm{c}}$ The position given is the estimated position of the center of mass of the IM Peg binary at epoch JD 2453403.0 (2005 February 1, 2005.08), the approximate midpoint of the GP-B science data. Along with the proper motion, the position is specified in the (J2000.0) coordinate system described in Section 3.1 and Paper III. This nearly inertial, extragalactic, coordinate system is closely tied to the International Celestial Reference Frame 2 (ICRF2; Fey et al. 2009).

${ }^{\mathrm{d}}$ As elsewhere, $\mu_{\alpha *}=\mu_{\alpha} \cos \delta$ (see Table 2, note a).

${ }^{\mathrm{e}}$ In our linear model, the orbital contribution to IM Peg's position at time $T$ is $A_{\mathrm{s} \alpha} \sin \left[2 \pi\left(T-T_{\mathrm{conj}}\right) / P\right]+A_{\mathrm{c} \alpha} \cos \left[2 \pi\left(T-T_{\mathrm{conj}}\right) / P\right]$ in $\alpha$ and $A_{\mathrm{s} \delta} \sin$ $\left[2 \pi\left(T-T_{\text {conj }}\right) / P\right]+A_{\mathrm{c} \delta} \cos \left[2 \pi\left(T-T_{\text {conj }}\right) / P\right]$ in $\delta$, where $P=24.64877 \mathrm{~d}$ is the (fixed) orbital period and $T_{\text {conj }}$ is the (fixed) time of conjunction, JD 2450342.905, adopted from Marsden et al. (2005).

${ }^{\mathrm{f}}$ See the text, Section 4.1 .

$\mathrm{g}$ The ratio of the minor axis to the major axis of the sky-projected orbit.

h See Paper VI for an illustration of the orbit geometry. The orbital motion on the sky is counterclockwise.

i Time of conjunction for the radio emitting region. The value shown is for the conjunction nearest the one with the primary in back, i.e., at its greatest distance from us, for the optical orbit of Marsden et al. (2005).

Solution 1 in that we also estimated the $\alpha$ and $\delta$ components of a constant proper acceleration. Solutions 3 and 4 are the two corresponding sets of results obtained by adding to our primary data set the position estimates we derived from our own reduction of the four sessions of IM Peg data obtained by Lestrade et al. (1995). Solution 5 differs from Solution 1 in that we excluded the nine positions derived from those VLBI images in which the radio emission from IM Peg was clearly resolved into more than one component. For Solution 6 we fit our nine-parameter model to the set of positions estimated using only AIPS processing (as opposed to "MA" processing, as described in Paper IV and discussed below) in the phasereferenced mapping steps of our data analysis. We now explain why these results lead us to conclude that the first set, Solution 1, provides reasonable estimates of IM Peg's parameters. The motivation for Solution 7 we defer until Section 4.3.2.

Comparison of the first two solutions in Table 2 reveals that inclusion of the acceleration term has at least two important effects. First, it increases the SSE of each of the two components of proper motion, $\mu_{\alpha *}$ and $\mu_{\delta}$, between three- and fourfold. The main cause of this increase is readily understood: because the 2005.08 reference epoch of the (time-varying) proper motion is less than half a year before the end of our $\sim 8.5 \mathrm{yr}$ data span, the estimates of $\mu_{\alpha *}$ and $\mu_{\delta}$ are, respectively, highly correlated (96\%) with those of $\dot{\mu}_{\alpha *}$ and $\dot{\mu}_{\delta}$. The increased SSEs follow from these high correlations. Second, inclusion of the acceleration causes changes in the proper-motion estimate. Indeed, the $\sim 0.3$ mas $\mathrm{yr}^{-1}(\sim 3 \sigma)$ change in $\mu_{\delta}$ exceeds the nominal accuracy requirement of our VLBI program. Therefore, we next justify why the proper-motion estimate from Solution 1 is clearly more appropriate for $G P-B$ than is the one from Solution 2.

Key support for this judgment is provided by Solutions 3 and 4, which differ from Solutions 1 and 2 in that we added to our set of IM Peg positions the four we determined from the VLBI observations of Lestrade et al. (1995). Comparison of 


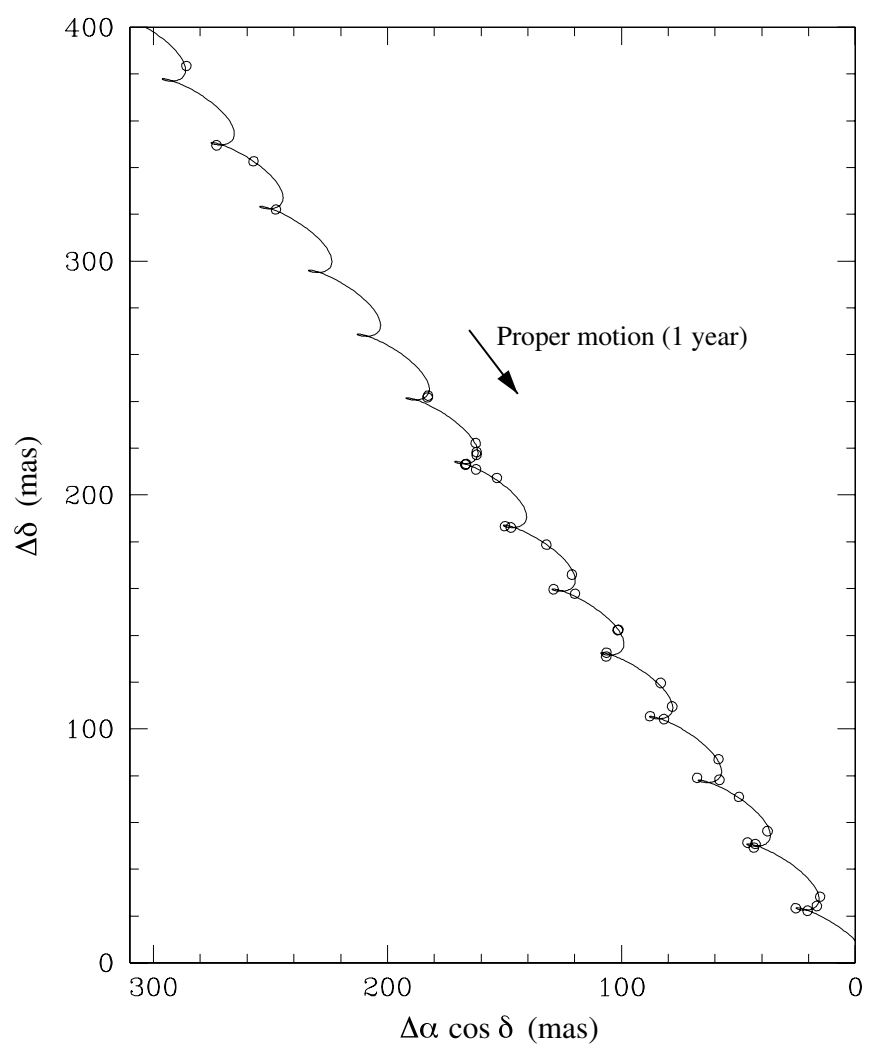

Figure 2. Our 39 VLBI-derived positions of IM Peg in Table 1 plotted to scale on the sky (with north at the top and east to the left). In this plot only, the coordinate origin is arbitrary. At this scale, our $\sim 0.1$ mas estimated measurement errors (see Section 3.2) are far smaller than the diameter of the position symbols. The plotted curve shows the astrometric fit of our chosen Solution 1 of Table 2, except that the orbital terms, only marginally visible at the scale of the figure, are excluded for clarity. At this scale, all data appear to be well fit, even though the first four data points (at upper left), spanning the years 1991-1994, were given no weight in this particular solution.

Solution 3 with Solution 1 reveals that, while the SSEs of $\mu_{\alpha *}$ and $\mu_{\delta}$ decrease by roughly one-third (as might be expected as a result of the $50 \%$ longer time span of the larger data set), the changes in the WLS estimates of $\mu_{\alpha *}$ and $\mu_{\delta}$ are each smaller than their respective SSEs in either of these solutions. In this sense the estimates from Solutions 1 and 3 are consistent with each other. On the other hand, comparison of Solution 4 with Solution 2 reveals that the added VLBI epochs not only reduce the SSEs of the proper-motion estimate, but also significantly alter that estimate, pushing it back toward that of Solution 1. The (previously) worrisome change in the estimate of $\mu_{\delta}$ introduced by solving for proper acceleration is reduced by a factor of 10 to a value much smaller than our nominal accuracy requirement. The high correlation between the proper-motion and properacceleration estimates (94\% in Solution 4) assures that there is also a corresponding effect on the proper-acceleration estimates. In fact, in Solution 4 neither $\dot{\mu}_{\alpha *}$ nor $\dot{\mu}_{\delta}$ is significantly different from zero. In addition, the model from Solution 2 fails to fit the (unweighted) 1991-1994 data, with its postfit $\delta$ residuals being up to 4 mas, which is about 10 times the rms postfit scatter in declination of the weighted points. In contrast, the model from Solution 1 fits the unweighted early data to within $\sim 1$ mas, as shown in the residual plot (Figure 3).

We draw three conclusions from our comparisons of these solutions: (1) Solution 2 gives a poor representation of the motion of IM Peg, (2) adoption of Solution 3 rather than Solution 1 as our nominal solution would have little effect on our proper- motion estimate, while decreasing its SSE by roughly $30 \%$, and (3) Solution 4 is less credible than Solution 1 since there is no independent evidence for nonzero $\dot{\mu}_{\alpha *}$ and $\dot{\mu}_{\delta}$. Furthermore, with other colleagues, we carried out an extensive, multifaceted observational search for a third stellar component. We found no credible evidence of any such component, ${ }^{6}$ allowing us to infer, under reasonable assumptions, that the probability of a detectable nonzero acceleration due to a third stellar component is less than $\sim 5 \%$. While the observational bounds on companions are difficult to quantify and summarize, this low probability is one of the computational results of a Bayesian statistical study (J. Chandler 2007, private communication) that combined these bounds with a range of plausible prior distributions of hypothetical third components with respect to mass, orbit parameters, luminosity, etc. Specifically, in light of the observational bounds on any companion of IM Peg, unless more than $80 \%$ of RS CVn binaries are assumed to have companions, there is a probability of only $\sim 5 \%$ that IM Peg in particular has one that causes a proper acceleration of the binary detectable at even the $1 \sigma$ level in Solution 4. (The separate principal result of this study is a $\sim 95 \%$-confidence statistical inference that the error in the guiding behavior of the GP$B$ spacecraft caused by light from any third component is a

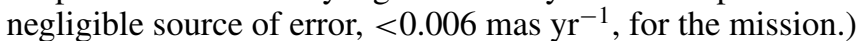

Table 2 also contains the results of two additional solutions made to test the sensitivity of our results to each of two other choices we made. As noted in Section 3.1 and Table 2, among the 35 VLBI position estimates that we used to obtain Solution 1 are nine from epochs at which our images of IM Peg resolve its radio emission into two or, in one instance, three components, separated by 1-2 mas (see Table 1; see also Figure 2 in Paper VII for images). Although the postfit residuals are neither noticeably larger or smaller at these nine than at the other epochs, we naturally were concerned that the VLBI positions at these epochs might be subject to systematic differences from those at the other epochs, in such a way as to significantly affect our results. We thus made Solution 5, with these nine epochs of data removed. Comparison of the estimates from this solution with those from Solution 1 is reassuring: our proper-motion estimate changes by

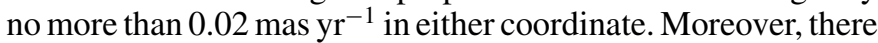
is no consistent change in the scatter of our postfit residuals: the rms of the $\alpha$ residuals decreases $6 \%$, but the rms in $\delta$ increases $4 \%$. Given these results, we conclude that Solution 1 need not be modified to account for the stellar radio emission's being at times resolved into spatially separated components. Solution 1 is also the most consistent with our general preference for the inclusion of all available high quality data, with uniform weighting of all sessions, since no other weighting is clearly justified. Of course, we could have exercised this preference still further by adopting Solution 3, obtained by fully weighting the four VLBIdetermined positions available from 1991 to 1994 . We did not do so for fear of underestimating the uncertainties associated with these positions. The smaller, more compact VLBI array used at these epochs makes the uncertainty of the corresponding position estimates larger and also more difficult to estimate.

\footnotetext{
6 The observational bound on the maximum optical brightness of undetected companions is, of course, wavelength dependent and also strongly dependent on angular separation. For example, based on our HST observations obtained with the $1042 \mathrm{~nm}$ WFPC2 filter, the minimum magnitude differences between the unresolved IM Peg binary and any third companion at angular separations on the sky of $0^{\prime \prime} 1,0^{\prime \prime} .5,1^{\prime \prime}$, and $5^{\prime \prime}$ are, respectively, about 5, 9, 11, and 16 mag. Observations through the $334 \mathrm{~nm}$ WFPC2 filter, as well as a wide variety of ground-based observations, listed in Paper I, yielded other useful magnitude limits, applicable to both larger and smaller angular separations.
} 

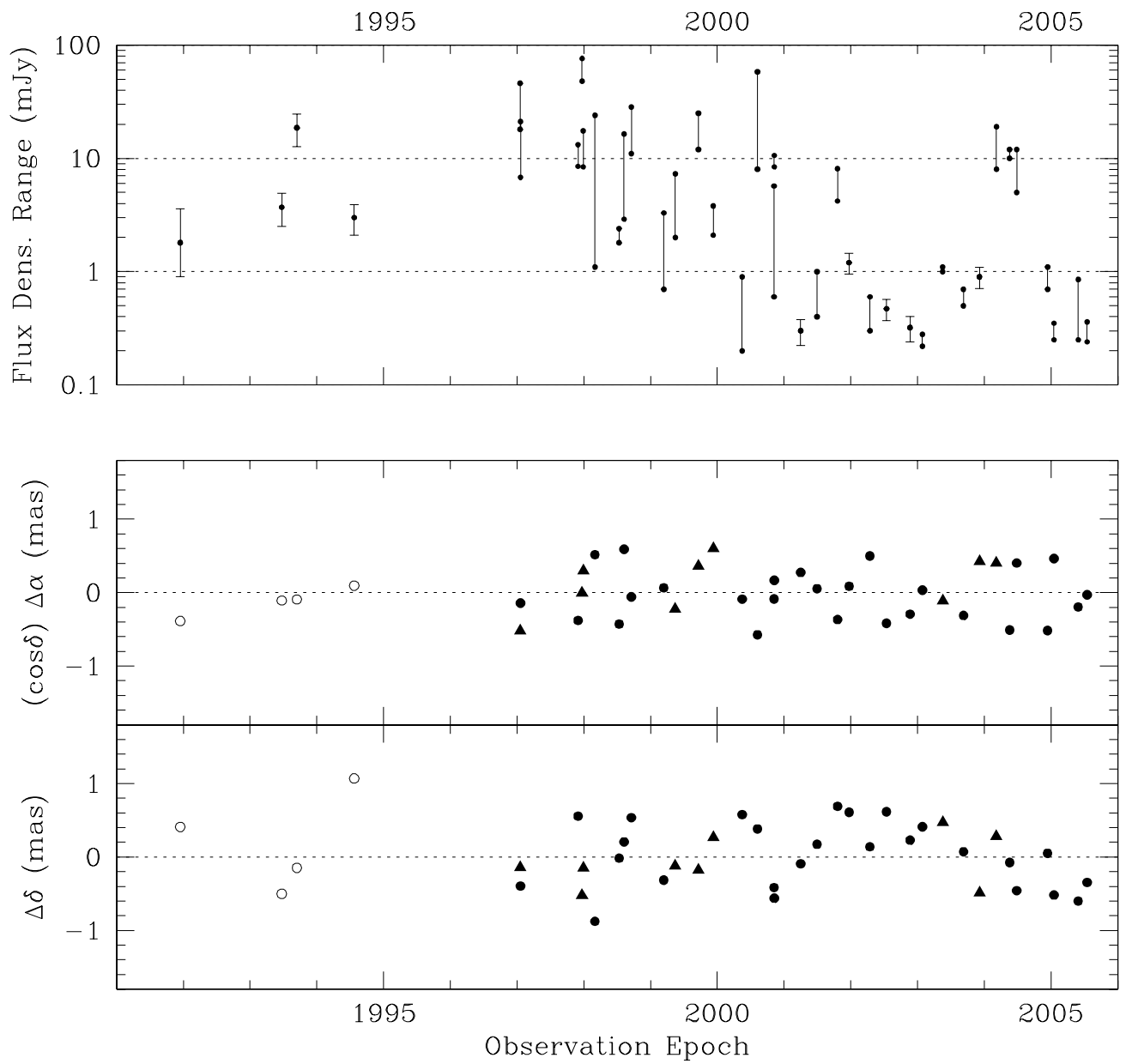

Figure 3. Upper panel shows flux densities for our 39 VLBI sessions. For 30 of these sessions, we show the range between the highest and lowest values of the flux density observed at the VLA, after smoothing with a boxcar window about 20 minutes wide. For the 2001 March session, there was insufficient signal at the VLA to clearly detect any change in the flux density, and so we plot just a single value. For the eight other sessions, the VLA was not used; for these sessions we also plot only a single flux density, the total flux density contained in our VLBI image of the emission. For each of these nine sessions, error bars indicate our estimated 70\% confidence interval for the total flux density, with allowance for amplitude calibration errors and the noise levels in our VLBI images. The two lower panels show the position residuals for all sessions for Solution 1 in Table 2 (see also Table 3). We plot unweighted points as open circles and weighted points as either closed circles or triangles, with each triangle indicating a position computed as the mean position of the two or three resolved peaks in the stellar radio image for that session (see the text, Section 3.1). We plot no error bars since we have no valid basis for assigning effective SEs to our VLBI position estimates for IM Peg, other than the scatter shown in this figure.

A conservative doubling of the position SEs, together with allowance for a plausible unknown common bias to these four positions relative to the others, would yield parameter estimates and SSEs closer to those of Solution 1 than to those of Solution 3. For simplicity, we choose to rely on Solution 1.

Solution 6 in Table 2 was made to explore a different aspect of our analysis, namely our use, as mentioned in Section 3.1, of VLBI phase calibrations derived from a Kalman-filter analysis of our VLBI data, rather than those computed within the basic AIPS package. To improve the accuracy of the phase models we produced with our Kalman-filter analysis, we normally included an ionospheric-delay model and employed updated, more accurate, Earth-orientation parameters and antenna positions than those originally contained in the AIPS data files. For Solution 6, we instead relied on AIPS calibrations alone. Consequently, for each parameter the size of the difference between the results from Solutions 1 and 6 provides a conservative, if rough, indication of the former result's uncertainty due to inaccuracies in our data-reduction models, or at least due to those inaccuracies that are not common to both of our phase-calibration procedures. Once again, the comparison between Solutions 1 and
6 is somewhat reassuring: our proper-motion estimate changes

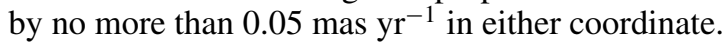

\subsection{Error Analysis \\ 4.3.1. Postfit Residuals}

For each solution in Table 2, the tabulated SSEs are those obtained directly from our WLS analysis. As discussed in Section 4.1, these SSEs would be unbiased estimates of the true SEs if all the errors in our measured positions were accounted for by our weighting scheme, which is based on the approximation that the position errors at all measurement epochs have identical and independent distributions. In this context, we regard any noiselike contribution to the offset on the sky between the position of the radio emission and that of the center of the primary as being a contribution to the measurement error. The causes of these (varying) offsets are not known, and so the best checks on their statistical properties are provided by examining our postfit residuals.

At first glance, the residuals in Figure 3 look like white noise, but there is a strong suggestion that the $\delta$ residuals, 


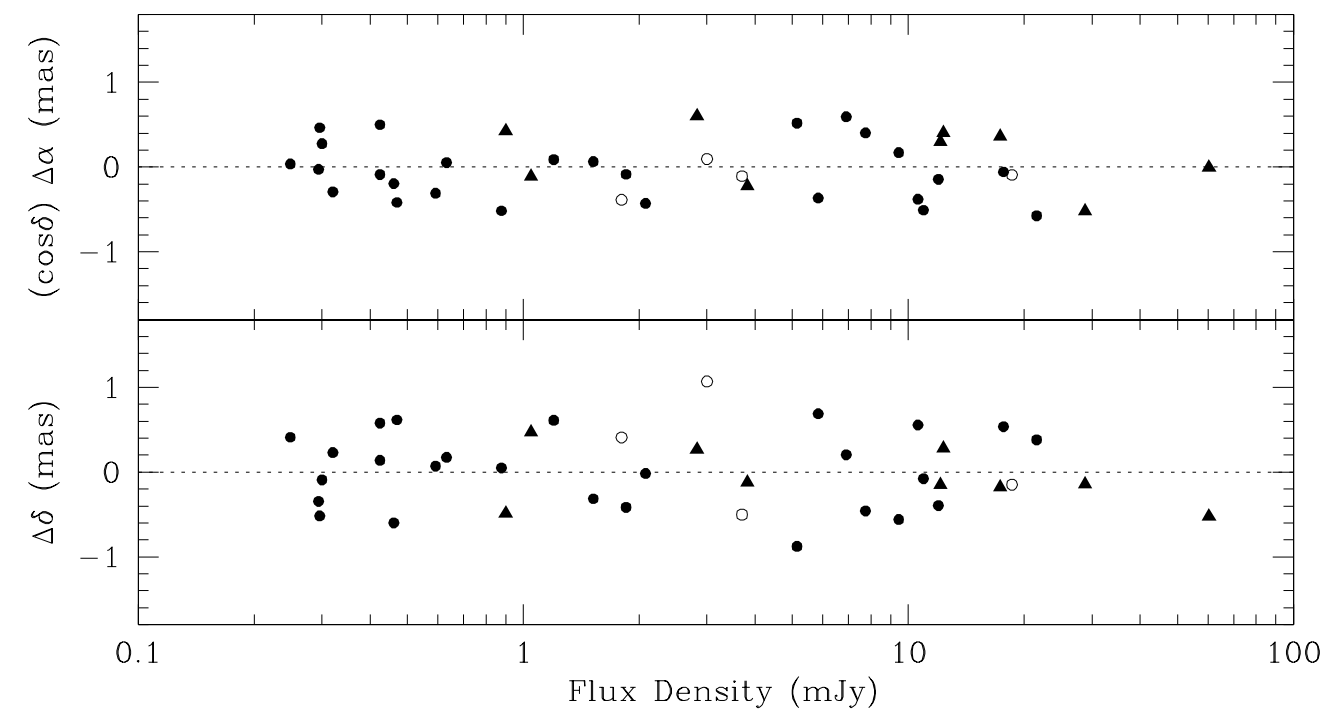

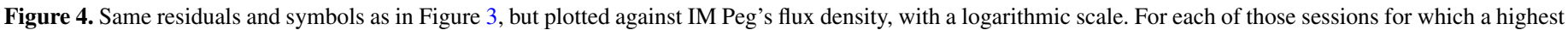

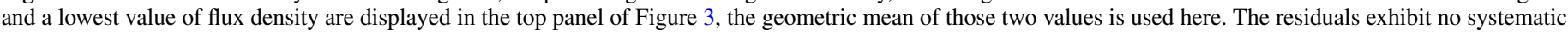
dependence on flux density.

at least for the later years, are not statistically independent between epochs separated by less than two years. Since such an autocorrelation would effectively reduce the number of independent measurements in our data set, it would tend to increase the true SSEs of our parameter estimates, and hence it is potentially important. We return to this issue below, after we complete our overview of the plots of our residuals.

The top panel of Figure 3 indicates the range of stellar flux density detected at the VLA during each VLBI session when that instrument observed IM Peg. Although the stellar flux density varied by a factor of ten or more during most years of our observations, it also exhibited an unmistakable downward trend over the 8.5 years of our observations. Consequently, if the variance of our position measurement errors (or any systematic bias in those measurements) were strongly correlated with flux density, our WLS proper-motion estimate would be subject to additional uncertainty that is not accounted for in our tabulated SSEs. To explore this possibility, we plot in Figure 4 our position residuals against flux density. No noticeable trend is seen, which suggests that any correlation between position residual and flux density is small and likely merely the result of random fluctuations within our modest-sized sample of measurements. There is also no evident relation between the amplitude of the scatter of the residuals and the measured flux density. Specifically, there is neither a significant linear trend nor any indication of larger-than-usual residuals being associated with either the highest or lowest flux densities observed. These observations bolster our decision to weight all our measured positions equally, regardless of the stellar flux density during the various sessions.

Similarly, our parallax estimate and its true SE could be adversely affected were the errors in the VLBI position measurements seasonally dependent. We therefore plot our postfit residuals against time of year in Figure 5. Here, too, the residuals appear to be merely noise with constant variance, with the possible exception of two one- or two-month-long parts of the year with several neighboring $\alpha$ residuals of the same sign. We conclude that the residuals do not justify increasing the SSE of our WLS estimate of IM Peg's parallax.
Just as the accuracy of our parallax estimate could be affected by a seasonal dependence in our VLBI position errors, our estimates of the orbit terms and their true SEs could be affected were our position errors dependent on orbit phase. Figure 6 shows our postfit position residuals plotted against orbit phase. Yet again, the residuals look like noise, with no systematic dependence on phase. There appears, therefore, to be no need to add any additional terms to our orbit model, or to allow for phase dependence of our measurement uncertainty. (Similarly, the plot of flux density against orbital phase, which is presented in Paper VII, shows no credible systematic relation between flux density and orbital phase.) We also computed Lomb-Scargle periodograms (Press et al. 1992) of our residuals. (For unevenly sampled time series like ours, such periodograms are more useful than Fourier transforms.) No peaks in the periodogram stood out as obviously significant. However, of the three (roughly comparable) highest peaks in the $\delta$ periodogram, the highest one (with a semi-amplitude corresponding to $\sim 0.36$ mas) occurred at period 8.16 days, which is within 0.06 days of exactly one-third of IM Peg's orbital period. It is unclear if this peak is significant, especially since there is no corresponding power excess in the $\alpha$ residuals. On the other hand, were the residuals white noise, the probability that the highest peak in the periodogram of either the $\alpha$ or the $\delta$ residuals would fall so close in frequency to either exactly two times or three times the reciprocal of the $\sim 24.65$ day orbit period is only $\sim 0.007$. Thus, the existence of the peak in the periodogram at 8.16 days suggests that the residuals contain at least some quasiperiodic signal. This suggestion is consistent with our understanding (see Section 3.2) that the residuals are largely due to variable offsets of the peak of the radio emission from the center of IM Peg's chromospherically active primary. Specifically, such offsets are likely related to the structure of the stellar magnetic field, which is, in turn, likely related to the photospheric spot distribution. Since we know from optical spectroscopy that the spot distribution varies only slowly, i.e., on timescales of months and years in a coordinate system that rotates with the same period as the near-circular orbit of the IM Peg binary (Berdyugina et al. 2000), presumably as a result of tidal locking, the offsets might well have a quasiperiodic 


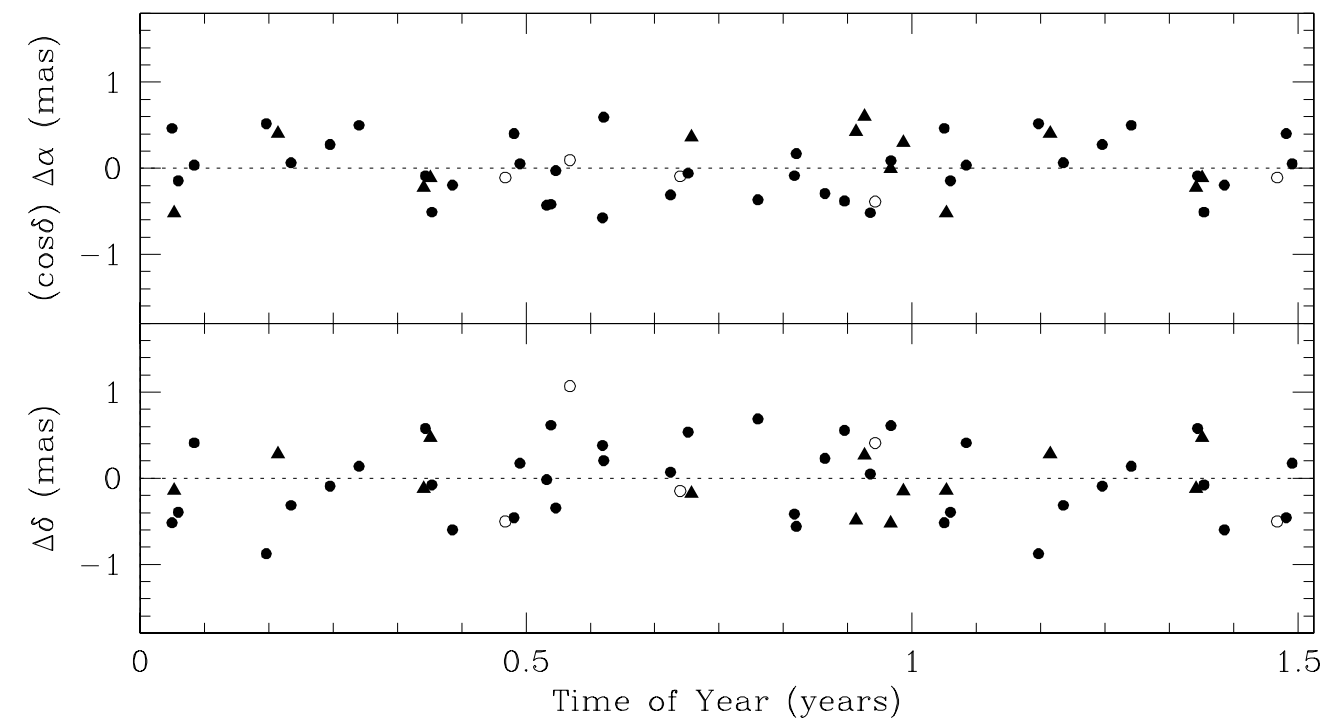

Figure 5. Same residuals and symbols as in Figure 3, but plotted against time of year in fractional years from J2000.0. To better demonstrate the lack of any trend from fall through winter, all residuals from the first half of any year are also plotted a second time shifted to the right by one year.

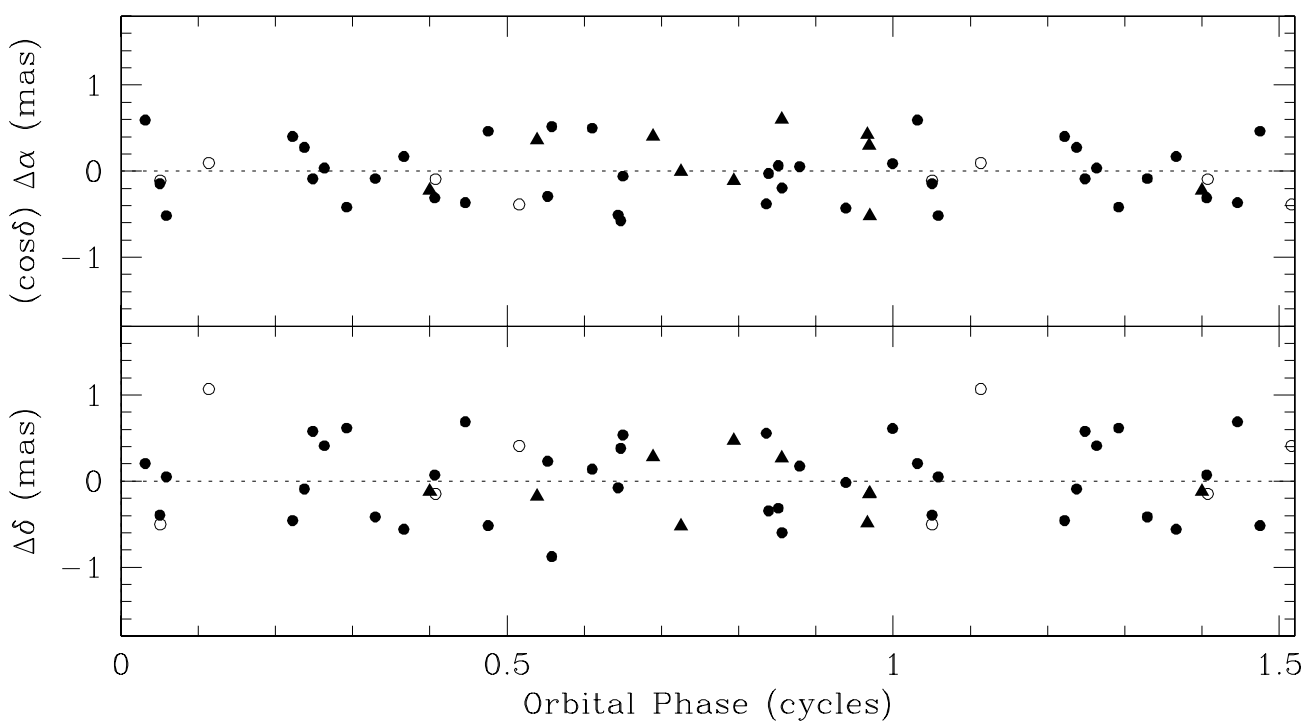

Figure 6. Same residuals and symbols as in Figure 3, but plotted against binary orbit phase in cycles. To better demonstrate the lack of any trend near the cycle boundary, all residuals from the first half of any cycle are also plotted a second time shifted to the right by one cycle.

component. Nevertheless, given the weakness of the statistical evidence for any periodic component in our residuals, we see no need to adjust the uncertainties of either our measured positions or the resulting parameter estimates to account for a possible dependence of those measurements on orbit phase.

In any case, the "orbit" we determine for the radio emission does not necessarily provide unbiased estimates of the size, shape, and phase of the orbit of the IM Peg primary. We discuss the strength of the correspondence in Section 6 and Paper VI, on account of its astrophysical significance. Such a correspondence, however, is not needed to meet the needs of the $G P-B$ mission. Solving for the orbital motion of the radio emission is a reasonable and demonstrably effective way of improving our fit to our measured positions and arguably, therefore, also the accuracy of our proper-motion and parallax estimates.

Could a non-Gaussian distribution of our position noise significantly affect the accuracy of our parameter estimates? Since this noise is in large part due to offsets of the peak of the stellar radio emission from the center of the IM Peg primary, or from some other nonstochastic mean position, there is no good reason to believe that this distribution is Gaussian. Nevertheless, because each of our WLS parameter estimates is calculated as a linear combination of the (two-dimensional) prefit residuals of our 35 measured positions with respect to our "initial-guess" model, the statistical distribution of each estimate will be approximately Gaussian, since each 70 term summation comes reasonably close to satisfying the conditions of the central limit theorems. Moreover, the SSEs of the parameter estimates yielded by the WLS approach do not depend on the shape of the measurement error distribution, but only on the measurement SEs.

\subsubsection{Epoch-to-epoch Error Correlation}

What about the apparently nonzero autocorrelation in the $\delta$ residuals, over time lags up to one or two years? The $\delta$ residuals in Figure 3 do not look like white noise, but it is far from clear what causes the pattern seen in them. 
It could arise from a deficiency of our astrometric model. The addition of an acceleration term to our model largely removes the below-zero mean from $\delta$ residuals for 2004 and 2005, but seven of the eight $\delta$ residuals from 2001 October through 2003 September remain positive, which suggests that this addition is not sufficient to leave only white noise in the residuals. Also, as noted earlier, the constant acceleration obtained from Solution 2 is not consistent with the IM Peg positions derived from the four earlier (1991-1994) VLBI sessions. Therefore, the addition of the acceleration term to our model seems unjustified. Neither does it appear that any reasonable enhancement of our orbit model (with, e.g., eccentricity, a third body, or anisotropic emission) could remove the largest systematic features in the residuals in Figure 3, features which each span about two years and include at least six epochs that are well distributed in phase. It is an even more open-ended task to rule out the possibility that the systematic residuals are caused by inaccuracies in our data-reduction scheme that could also contribute important systematic errors to our astrometric parameter estimates. What we can say is that, first, we cannot identify any such inaccuracies that could plausibly explain our $\delta$ residuals, and, second, the lack of comparably large and systematic residuals in our fits for the relative positions of our three reference sources, despite their larger separations on the sky, ${ }^{7}$ argues strongly that the origin of these features lies not in modeling errors (such as errors in our corrections for the effects of the ionosphere), but rather in changes in the physical location of the IM Peg radio emission region. The statistical properties and possible physical interpretations of these changes are explored in Papers VI and VII.

Furthermore, it is not unreasonable that the motion of that radio emission about a mean orbit fit to its positions would appear to be noise with a nonzero autocorrelation function. The major obstacle to our accounting for the effects of such systematic residuals with a suitable noise model is that we lack adequate prior knowledge of the autocorrelation function. It could plausibly be monotonic, periodic, or quasiperiodic, though the periodogram analysis of the residuals (described above) failed to identify any significant periodicity. Even for monotonically decreasing functions of time separation, there is inadequate constraint on the appropriate functional form. The inadequacy is worsened by our having just a few effectively independent samples of any noise process whose autocorrelation width is one or two years. We therefore collaborated with Jingchen Liu and Xiao-Li Meng of Harvard's Department of Statistics on statistical exploration and Bayesian analyses of our position estimates. These analyses (Liu 2008) revealed that allowance for the autocorrelation could increase those SSEs by a factor of two or so, depending, of course, on the prior probability distributions we adopt for the parameters describing the autocorrelation. Rather than discussing and interpreting those uncertain results, we take a simpler approach here.

Suppose we were to assume that, for the purpose of estimating IM Peg's proper motion, we can regard our observations as yielding only one independent measurement every two years. Given that we obtained about four positions per year, the uncertainty of our WLS proper-motion estimate would be increased by a factor of up to $\sqrt{8}$, but such a "worst-case" increase almost

\footnotetext{
7 During the above-mentioned period 2001 October through 2003 September, in which the IM Peg positions have a mean residual of $\sim+0.5$ mas in declination, the mean offsets of the three reference sources relative to each other are all less than 0.2 mas in any direction (as can be inferred from the position residuals plotted in Figure 6 of Paper III).
}

surely overestimates the effect of the autocorrelation, given that there is no two-year subset of our data during which the mean $\alpha$ or $\delta$ residual was larger than the rms difference between consecutively measured position coordinates. Consequently, to estimate the statistical contribution to the total SE of our proper-motion estimate, we reduce the worst-case factor of $\sqrt{8}$ to a factor of two. Although this choice is clearly somewhat arbitrary, we believe that any significantly larger factor would be unrealistically conservative.

We can check the appropriateness of our choice of a factor of two by making trial solutions in which we remove an interval of data that might be affected by a relatively large value of some noiselike but autocorrelated offset. Solution 7 in Table 2 is one such solution. We obtained it by excluding the final five VLBI positions, which are those whose residuals exhibit (in Figure 3) the most clearly systematic and one-sided offsets from the model yielded by Solution 1 . The resulting proper-motion estimate differs from that from Solution 1 by 0.07 mas $\mathrm{yr}^{-1}$

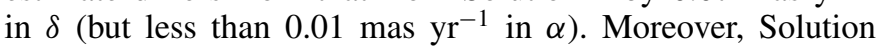
7 underestimates the mean declination of the four unweighted 1991-1994 VLBI positions by 0.6 mas, suggesting that this solution is inferior to Solution 1, which underestimates their mean declination by only 0.2 mas. Further, we can surmise from inspection of Figure 3 that the omission of no other group of five consecutive positions would cause even this large a change. Thus, we consider Solution 7 to be good evidence that doubling

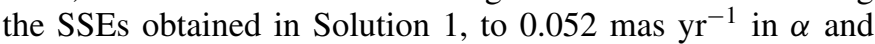
0.059 mas $\mathrm{yr}^{-1}$ in $\delta$, gives reasonably conservative values for SSEs with adequate allowance for the possible contributions of errors that are correlated between different epochs.

We also increase our initial estimates of the SSEs in IM Peg's position coordinates at the reference epoch by the same somewhat arbitrary factor, since those estimates, too, would be strongly affected by any autocorrelation spanning a year or two. The other parameters in our nine-parameter solutions govern terms in our astrometric model that are periodic with a period of either $1 \mathrm{yr}$ or $\sim 24.65$ days. If autocorrelation of the noise in our position estimates is consistently positive for time differences less than a year, the uncertainties in our estimates of these parameters would be smaller, not larger, than they would be without such autocorrelation. Thus, there is no reason to increase our SSE estimates for these other parameters.

\subsubsection{Systematic Errors}

We turn now to other contributions to our uncertainty: propagation delays, inadequately mapped source structure, and inaccuracies in the parameter values used in the reduction of our data, such as inaccuracies in Earth-orientation parameters and antenna positions. Based on the observed stability of the relative positions of our three reference sources, we place upper bounds on all errors that are not intrinsic to IM Peg. (The more direct approach, summing in quadrature the estimated SEs due to the individual known sources of systematic error, would be less comprehensive and hence less reliable.)

What, in particular, can we learn from our reference-source results? In Paper III we estimate the relative proper motions between the chosen reference points in our three reference sources. Allowing for $1 \sigma$ uncertainties, we find that the " $\mathrm{C} 1$ " core component of 3C 454.3 moves with respect to each of the other two sources by less than 0.04 mas $\mathrm{yr}^{-1}$ in each coordinate. Since the separation from 3C 454.3 of one of these sources is twice that of IM Peg, and that of the other is five times greater, these results suggest that all the sky-separation- 
dependent error sources listed above, including ionospheric

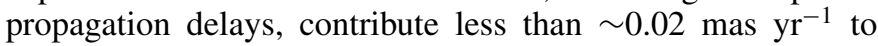
the SE of IM Peg's proper motion. Moreover, as discussed in Paper III, due to the established or inferred cosmological distance of each of these reference sources, we expect that the true proper motion of their radio cores is smaller still. It would thus require very unlikely cancellations for the above

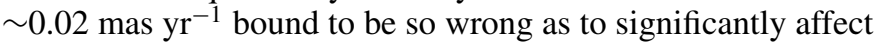
our value for the total SE of IM Peg's proper motion. A similar argument can be made concerning the errors associated with the individual radio sources, including those associated with the C1 core component of 3C 454.3. In Paper III, over the span 1998.71-2005.54, we determine upper bounds on C1's proper

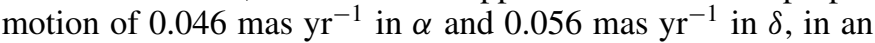
extragalactic reference frame closely related to the International Celestial Reference Frame 2 (ICRF2; Fey et al. 2009). The sizes of the observed differential proper motions among our reference sources quoted above suggest that these upper bounds are conservative, since part of the relative proper motions is likely due to motions of the brightness peaks of B2250+194 and B2252+172. We therefore adopt these last upper bounds as the total contributions to the SE of the corresponding coordinates of our estimate of IM Peg's proper motion due to all uncertainties not intrinsic to IM Peg.

We must also allow for the possibility of systematic changes in the location of the radio emission relative to the IM Peg primary. The radio emission from IM Peg is fairly typical of that from other RS CVn spectroscopic binaries, which, like IM Peg, usually contain a chromospherically active, cool giant primary and an inactive secondary (Guinan \& Giménez 1993). Thus, there is a good reason to infer that IM Peg's radio emission is closely associated, both causally and spatially, with the primary. Indeed, as discussed below in Section 6, the orbit we determine for the radio emission is consistent with that of the primary. However, that consistency does not rule out the existence, during the span of our observations, of a significant trend in the spatial offset between the peak of the radio emission and the center of the primary. In particular, we must consider the possible effect of a slow evolution of that star's magnetic field, since such evolution might be expected if the star has a multiyear magnetic cycle. The strong downward trend in the flux density of IM Peg's radio emission (see Figure 3 ) is, in itself, strong evidence of some kind of evolution of the radio emission region. Thus, we must make some allowance for the possibility that the position of the radio emission relative to the stellar components of the binary has a nonzero trend. Since the rotation period of the primary is approximately equal to the binary orbital period (as noted above in Section 4.3.1), one can expect that any offset that persists for an orbit period or longer between the center of the radio-emitting region and the center of the primary would also corotate with the binary, and hence that the offset's equatorial component would largely average out in a nonrotating frame. On the other hand, the component normal to the equatorial plane would be identical in the rotating and inertial frames, and so could plausibly exhibit a long-term trend as seen from Earth. The key question is: how large could that trend plausibly have been over our observing span?

Since we have no way to measure any such trend, the best we can do is to conservatively estimate a plausible rms magnitude for it. Three lines of reasoning suggest that the net change, over the span of our observations, of the mean offset described above is likely too small to contribute a major source of error. In our images of IM Peg, the mean apparent extent of the emission region is $1.4 \pm 0.4$ mas (see Paper VII), and only at 6 of our 35 epochs did the length exceed 2 mas, with the maximum value being 3.3 mas (on 2001 January 15). Also, within any individual session, we never detected motions of the brightness peak exceeding 1 mas. More importantly, using the empirical model of the spatial distribution of the brightness peaks relative to the IM Peg primary that we develop in Paper VI, we find that $2 / 3$ of them occur at a distance from the center of the primary that subtends no more than 0.8 mas. Thus, we conclude that even during individual VLBI sessions the offset of our VLBI position from the primary is unlikely to exceed $\sim 1$ mas.

In addition, while there was a strong (though "noisy") downward trend in the stellar radio flux density over the span of our observations, there was no clear corresponding trend in the evolution of the shape of the emission region. This lack of a clear trend in shape adds to our confidence that, over the $8.5 \mathrm{yr}$ span of our VLBI observations, the net motion of the mean position of the emission with respect to the IM Peg primary did not vary by as much as $\sim 1$ mas. Consequently, it is implausible that mean rate of relative motion over that span exceeded $\sim 0.12$ mas yr$^{-1}$. Even this rate of angular motion seems implausibly large for an rms value, for two reasons. Given the above maximum offset, any stellar activity cycle with a period significantly different from about twice the length of our observations would result in significantly lower drift rates. Secondly, although our lack of knowledge of the uncertainties of the four radio position estimates we obtained from VLBI observations between 1991 and 1994 led us to omit those estimates from our chosen Solution 1, they do provide at least some evidence that the timescale of any systematic drift is more than $\sim 14 \mathrm{yr}$. We thus believe that the rate corresponding to a shift of one stellar

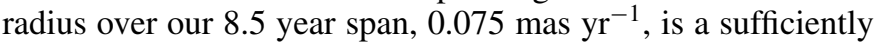
conservative estimate for the rms drift rate. Since the projection on the sky of the orbit normal is along P.A. $=130.5 \pm 8.6$, we allow for an rms systematic error contribution in our proper-

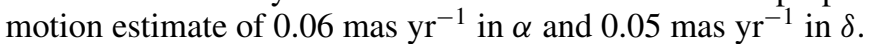

Systematic errors could also affect our estimates for other parameters. Our position estimate for the center of mass of the binary at any given reference epoch could be biased due to the type of systematic offset discussed directly above. Indeed, as discussed in Paper VI, such a bias may well be caused by the ongoing partial occultation by the primary of the radio emission region that we infer surrounds it. The inclination of the spin axis to the plane of the sky (discussed in Sections 5 and 6), together with the observed elongation of the scatter of our VLBI residuals (discussed in Paper VI), breaks the symmetry that might otherwise have led us to believe that the offset between the radio emission and the center of the binary system would average out over time. Thus, we must make some estimate of the expected value and the uncertainty of the resulting error contributed to the position we estimate for the binary center of mass at the mean epoch of our VLBI observations, 2001.29. Because we have no reliable quantitative model of this contribution, we are forced to make somewhat arbitrary choices for its mean value and uncertainty. We do so in light of the considerations stated above in regard to the mean rate of change of the error during our span of VLBI observations. For simplicity, we take the expected value for the bias to be zero and its rms error to be one-half the angular radius of the primary, directed along the sky projection of our inferred direction for the normal to the binary orbital plane. To calculate the rms systematic position error at any other epoch, we assume that this error at mean epoch 2001.29 is statistically 
independent of any related systematic error in our proper-motion estimate. Thus, to our error at 2001.29 we add in quadrature the product of the rms drift rate estimated above and the time difference between the other epoch and 2001.29. In any case, the $G P-B$ relativity tests have virtually no sensitivity to any possible milliarcsecond-scale constant bias in our estimate of the position of IM Peg; we discuss the bias only to facilitate the future use of our position estimate for some other purpose, such as for helping to tie the reference frame of a stellar position catalog to that of an extragalactic radio source catalog.

In our parallax estimate, systematic errors could occur if our position estimates are subject to seasonally dependent errors. Indeed, it is plausible that inaccuracies in our models of the troposphere and ionosphere contribute such seasonally dependent errors to our position measurements. Thus, we need an estimate of the size of the resulting parallax errors, or some bound on it. We pursued three separate approaches to this goal.

First, because VLBI observations made during more humid, warmer months tend to suffer larger atmospheric effects, we obtained an additional astrometric solution (not tabulated) after excluding the positions from such observations. Since all but one of our antennas are in the Northern Hemisphere, we excluded all observations from April through September. Unfortunately, this solution (and also a complementary solution based on data obtained only from April through September) yielded a parallax SSE three times (and four times) larger than did the solution using all the data. Consequently, even though the resulting changes in our parallax estimate were up to 4.4 times the SSE of the earlier estimate, they were each less than 1.5 times the SSE of the corresponding new estimate, and hence not truly meaningful.

Second, we exploited the tendency of atmospheric errors to more strongly affect differential $\delta$ estimates than differential $\alpha$ estimates, for sources observed over a range of hour angles spanning the central meridian, as was the case at the central sites of our VLBI array. This tendency is particularly strong in the relevant case of source pairs separated mainly in $\delta$ (see, e.g., Pradel et al. 2006). A fit to our $\alpha$ data alone yielded a parallax estimate that differed from our chosen estimate by only 0.045 mas, with an SSE only 20\% larger than that of the fit to all the data. This result, coupled with those in Figure 5, suggests that systematic error did not significantly degrade our estimated $\delta$ coordinates more than our $\alpha$ coordinates. We thus infer that our atmospheric models likely did not significantly degrade our parallax estimate.

Finally, we derived a third and strongest upper bound on the plausible size of any systematic error in our parallax estimate by using our VLBI data to estimate the relative parallax between B2250+194 and 3C 454.3, and taking advantage of the fact that their great distances from the solar system ensure that their true parallaxes are undetectably small. To obtain a close analog to the systematic error in our stellar parallax estimate, we fit to the 35 relative positions we estimated for B2250+194 with respect to $3 \mathrm{C} 454.3$ the same nine-parameter model used to model the motions of IM Peg. We also gave those differential positions the same uniform SEs that we use for IM Peg. The resulting parallax estimate for $\mathrm{B} 2250+194$ is $-0.032 \pm 0.074$ mas. Alternatively, if we rescale the position SEs so that the value of $\chi^{2}$ per degree of freedom of the resulting residuals is unity, the SSE of the parallax estimate falls to 0.026 mas. Either way, the result suggests that the systematic error in the B2250+194 parallax estimate is smaller than the 0.074 mas SSE of our IM Peg results. Moreover, to estimate the size of the systematic error
Table 4

Correlation Matrix for the IM Peg Parameter Estimates

\begin{tabular}{lrrrrrrrrr}
\hline \hline Parameter & \multicolumn{1}{c}{$\alpha$} & \multicolumn{1}{c}{$\delta$} & \multicolumn{1}{c}{$\mu_{\alpha *}$} & $\mu_{\delta}$ & Parallax & $A_{\mathrm{s} \alpha}$ & $A_{\mathrm{s} \delta}$ & $A_{\mathrm{c} \alpha}$ & $A_{\mathrm{c} \delta}$ \\
\hline$\alpha$ & 1.00 & & & & & & & & \\
$\delta$ & -0.30 & 1.00 & & & & & & & \\
$\mu_{\alpha *}$ & $\mathbf{0 . 8 3}^{\mathrm{a}}$ & -0.25 & 1.00 & & & & & & \\
$\mu_{\delta}$ & -0.25 & $\mathbf{0 . 8 3}^{\mathrm{a}}$ & -0.29 & 1.00 & & & & & \\
Parallax & -0.12 & -0.05 & -0.19 & -0.07 & 1.00 & & & & \\
$A_{\mathrm{s} \alpha}$ & 0.03 & -0.01 & -0.06 & 0.01 & 0.05 & 1.00 & & & \\
$A_{\mathrm{s} \delta}$ & -0.01 & 0.03 & 0.02 & -0.06 & 0.01 & -0.31 & 1.00 & & \\
$A_{\mathrm{c} \alpha}$ & 0.12 & -0.04 & 0.15 & -0.05 & 0.02 & 0.12 & -0.04 & 1.00 \\
$A_{\mathrm{c} \delta}$ & -0.04 & 0.12 & -0.06 & 0.15 & 0.04 & -0.03 & 0.12 & -0.31 & 1.00 \\
\hline
\end{tabular}

Note. ${ }^{a}$ The two largest table entries are highlighted in bold.

in our stellar parallax, we should take into account that the separation of IM Peg from 3C 454.3 is fivefold smaller than that of B2250+194 from 3C 454.3. Since virtually all of the seasonally dependent contributions to our measurement errors can be expected to scale with (vector) separation on the sky, we estimate that the systematic contribution to the SE of our stellar parallax value is, at most, one fifth of its 0.074 mas SSE. We thus take 0.015 mas as a plausible upper bound on the rms systematic error in our stellar parallax estimate. This bound implies that systematic error makes a negligible contribution to the total SE of the estimate.

The above-mentioned uniformly weighted fit to our differential reference-source positions also provides a test for systematic errors in our estimates of the four sinusoidal terms in our model for the IM Peg radio emission. As would be expected in the absence of significant systematic error, none of the four "test" estimates differs significantly from zero; the largest in absolute value is only 0.05 mas (and only 1.2 times its SSE). In light of this test and all the others mentioned previously, we believe that our error allowances are consistent with our data and adequate to estimate the true uncertainties of our results.

\section{FINAL RESULTS}

We present in Table 3 our final results from Solution 1 for all nine astrometric parameters that define our model, using each of the following epochs: 2005.08 (2005 February 1, the approximate midpoint of the $G P-B$ science data), 2001.29 (the mean epoch of the 1997-2005 VLBI data, 1991.25 (the epoch of the Hipparcos Catalogue), and 2000.0. We present, too, an alternative parameterization of the projected orbit: length of its semimajor axis, position angle of the major axis (which is also that of the node in the plane of the sky), axial ratio, and time of conjunction (the one nearest the time of conjunction estimated by Marsden et al. 2005). The table also contains the SSEs from the WLS fit and our estimated total SEs. These latter contain allowances for the apparent autocorrelation in the VLBI position errors and for other possible systematic errors, with their variances summed under the reasonable assumption that the systematic errors are independent of each other.

The correlations of the parameter estimates from our WLS fit are given in Table 4. The high correlations among the estimates of position and proper motion are a consequence of the displacement of the reference epoch from the mean epoch of our data. (At the mean epoch of our VLBI data, 2001.29, the correlations are only -0.024 for the $\delta$ components and -0.015 for the $\alpha$ components.) In contrast, the dominant cause of the correlations between the $\alpha$ and $\delta$ components of these terms 
Table 5

Comparison with Other Results

\begin{tabular}{lcccc}
\hline \hline Result & Epoch & \multicolumn{2}{c}{ Proper Motion } & \multirow{2}{*}{\begin{tabular}{c} 
Parallax \\
\cline { 2 - 4 }
\end{tabular}} \\
\cline { 2 - 4 } & & $\begin{array}{c}\mu_{\alpha *} \\
\left(\mathrm{mas} \mathrm{yr}^{-1}\right)\end{array}$ & $\begin{array}{c}\mu_{\delta} \\
\left(\mathrm{mas} \mathrm{yr}^{-1}\right)\end{array}$ & $\begin{array}{c}\pi \\
(\mathrm{mas})\end{array}$ \\
\hline This paper & 2001.29 & $-20.83 \pm 0.09$ & $-27.27 \pm 0.09$ & $10.37 \pm 0.07$ \\
Lestrade et al. (1999) & 1992.92 & $-20.59 \pm 0.46$ & $-27.53 \pm 0.40$ & $10.28 \pm 0.62$ \\
Hipparcos Catalogue & 1991.25 & $-20.97 \pm 0.61$ & $-27.59 \pm 0.57$ & $10.33 \pm 0.76$ \\
van Leeuwen (2008) & 1991.25 & $-20.73 \pm 0.28$ & $-27.75 \pm 0.27$ & $11.17 \pm 0.33$ \\
Tycho-2 Catalogue & $\sim 1960^{\mathrm{a}}$ & $-21.4 \pm 1.0$ & $-26.3 \pm 1.0$ & $\ldots$ \\
\hline
\end{tabular}

Note. ${ }^{\text {a }}$ We assign this approximate epoch because, for our own similar optical data set, for both $\alpha$ and $\delta$ the correlation between our proper-motion estimate and our proper-acceleration estimate vanishes near this epoch. Consequently, when we also estimate IM Peg's proper acceleration from the optical data, our proper-motion SSEs are smallest near this epoch.

and also between those of the orbit terms is the correlation at each epoch of the noiselike errors in $\alpha$ and $\delta$, whose value we set at -0.314 based on the iterative procedure described in Section 4.1. The relatively small magnitude of the correlations among the other estimates is the result of our successful efforts to schedule our observations at epochs well distributed over orbit phase and season of the year. We do not adjust these correlations to account for systematic errors, primarily because we have insufficient knowledge of the correlations between the $\alpha$ and $\delta$ components of those errors.

\section{COMPARISON OF RESULTS WITH PREVIOUS ESTIMATES}

Our results are compared with those from several other sources in Table 5. Our results for proper motion and parallax are consistent with those of Lestrade et al. (1999) within their (larger) SEs. Both of these sets of results are in agreement with the corresponding values in the Hipparcos Catalogue (ESA 1997) to within the latter's SEs, which are slightly larger than those of Lestrade et al. However, there is some disagreement with the IM Peg results of the new Hipparcos reduction of van Leeuwen (2007, 2008). For IM Peg's proper motion, the errors yielded by the new reduction are about threefold larger than ours, although $30 \%-50 \%$ smaller than those of Lestrade et al. Our results agree with those in van Leeuwen (2008) in $\mu_{\alpha *}$ to within the combined SE, but disagree by 1.6 times the combined SE in $\mu_{\delta}$ and 2.4 times the combined SE in parallax. The astrometric orbit of the binary, not allowed for in either of the two Hipparcos analyses, could be an additional source of systematic error in the Hipparcos parallax values (and also in the results of Lestrade et al.). In any case, the 0.48 mas $\mathrm{yr}^{-1}$ disagreement in $\mu_{\delta}$ is of no consequence for the measurement of the (north-south) relativistic geodetic effect achieved by the $G P-B$ mission, $-6601.8 \pm 18.3$ mas (Everitt et al. 2011). Furthermore, the disagreement in $\mu_{\delta}$ estimates, like that in the parallax estimates, has no direct or significant effect on $G P-B$ 's measurement of the smaller frame-dragging effect, which manifests itself as a purely eastward drift of $G P$ $B$ 's onboard gyros with respect to IM Peg; this result was $-37.2 \pm 7.2$ mas (Everitt et al. 2011).

The comparison of our (VLBI) $\mu_{\alpha *}$ and $\mu_{\delta}$ results with the corresponding Hipparcos (optical) results also provides a check on the size of the systematic error due to the drift of IM Peg's radio emission with respect to the center of mass of the binary system. However, that check is not particularly useful to us, since

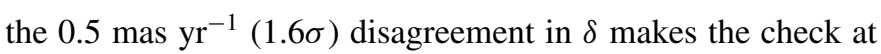
least tenfold less precise than the bound for which we argued in Section 4.3.

We also looked for evidence of proper acceleration in a set of optical astrometric positions that were collected and rotated onto a common reference frame for the purpose of computing the proper motions in the Tycho-2 Catalogue (Høg et al. 2000). We performed two WLS fits to the 14 optical IM Peg positions given to us by N. Zacharias (2006, private communication), which spanned 1897-1991. (The final year contains the positions from the Hipparcos and Tycho observations, which are far more accurate than the others, most of which have 100-300 mas SEs.) Our four-parameter fit (for position and proper motion only) yields a proper motion in agreement with the Tycho-2 value to within 0.13 mas $\mathrm{yr}^{-1}$ in each coordinate, which is a small fraction of the estimated $\sim 2.5$ mas $\mathrm{yr}^{-1}$ precision of the Tycho-2 proper motions. (The results are not identical due, at least in part, to differences between the sets of optical positions included in the two reductions.) More importantly, when we also estimate a proper acceleration, we find it to be consistent with zero to within the $\sim 0.09$ mas $\mathrm{yr}^{-2}$ SSE of each coordinate of that estimate. This result rules out the (unlikely) possibility that, on account of such an acceleration, the Hipparcos proper motion might be greatly in error at the $G P-B$ epoch, nearly 14 years later than the Hipparcos mean epoch. Combined with the Hipparcos proper motion, the acceleration estimate thus provides a completely independent and purely optical check on our main proper-motion estimate. The uncertainty of this check

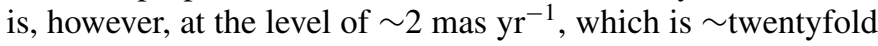
larger than that of our own estimate.

The comparison of our IM Peg position at epoch 1991.25 with the Hipparcos position at the same epoch reveals a $\sim 2.4$ mas discrepancy, almost purely in $\delta$, that is more than twice the combined SEs. Obtaining our result at that epoch required an extrapolation (back in time) of nearly six years from the start of our main data set. Considering the possible difficulties involved in ensuring that the two celestial reference frames are truly aligned to adequate accuracy, and also the problems generally associated with the extrapolation of data, we will not attempt to interpret the discrepancy, or even judge if it is truly significant.

Unlike the optical results discussed above, spectroscopic investigations of IM Peg yield three of the estimated parameters of our VLBI-derived orbit. Marsden et al. (2005) estimate the parameters of circular orbits (by assumption, based on the $0.006 \pm 0.007$ estimate of eccentricity by Berdyugina et al. 1999) for both stellar components. Combining the spectroscopic results with estimates of the orbital inclination and parallax of the system, we can compute the angular sizes of these orbits. Berdyugina et al. determine the orbital inclination to lie between $65^{\circ}$ and $80^{\circ}$, while Lebach et al. (1999) found a lower bound of $\sim 55^{\circ}$. In comparison, the $0.30 \pm 0.13$ axial ratio of our VLBIderived projected orbit (Table 3 ), corresponds to an inclination of $73^{\circ} \pm 8^{\circ}$. The good agreement is consistent with the radio orbit having the same inclination as the optical orbit, as would be expected under the plausible assumption that the effects of any anisotropy of the emission process or any partial stellar occultation of the emission region do not significantly affect the shape of our VLBI-derived orbit. Combining our inclination and parallax estimates with Marsden et al.'s $a$ sin $i$ estimate for each component leads to projected orbit semimajor axes of $0.84 \pm 0.03$ mas for the primary and $1.53 \pm 0.06$ mas for the secondary. Thus, the $0.89 \pm 0.09$ mas semimajor axis of our radio orbit agrees with that of the primary, but differs 
significantly (by six times the combined SE) from that of the secondary. This result is consistent with our expectation that the radio-emitting region is more closely associated with IM Peg's primary than with its secondary. This result is also qualitatively analogous to the finding of Lestrade et al. (1993) for the Algol system: the motion of its radio emission on the sky is consistent with the optically determined orbital parameters of the active evolved star of the close binary. We can also compare the time of conjunction implied by our orbit with the corresponding time found by Marsden et al. Our estimate is $0.34 \pm 0.44 \mathrm{~d}$ earlier. Thus, our orbit for the radio emission is not only the same size as that of the primary, but also in the same phase, to within our SE. In light of our estimates of parallax and orbit size, we can set a $0.78 \mathrm{~d} 1 \sigma$ upper bound on the phase difference, which corresponds to a physical distance of $3.3 R_{\odot}$ between the center of the primary and the mean position of the radio emission. The radius of the primary, as estimated by Berdyugina et al. (1999), is $13.3 \pm 0.6 R_{\odot}$. Consequently, the center of the radio emission is on average offset in phase from the estimated center of the primary by no more than about one-fourth of the latter's radius.

\section{CONCLUSIONS}

1. From our series of 35 VLBI sessions spanning from 1997 January to 2005 July, we obtained weighted least-squares estimates for the position, proper motion, parallax, and skyprojected circular orbit of the radio emission from IM Peg (see Table 3).

2. The accuracy of these parameter estimates is limited primarily by the noiselike scatter in our VLBI position measurements for IM Peg. This scatter is not caused primarily by measurement error, but rather by apparently random offsets of the brightness peaks of the stellar emission from any Keplerian orbit that can be fit to these peaks.

3. For IM Peg's proper-motion parameters, and for those specifying its center-of-mass position at epoch, we allow for increased statistical error due to the apparent correlations in the position residuals for sessions separated by less than 1 yr. We also allow for a possible nonzero mean offset and systematic drift of the stellar radio emission with respect to the center of mass of the binary.

4. Our parameter estimates for IM Peg's proper motion and parallax are each consistent with previous optical and VLBI estimates to within the appropriate combined standard errors, with two exceptions: (1) the $\delta$ component of our proper motion and that of van Leeuwen (2008) disagrees

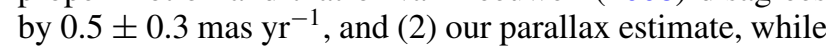
consistent with that of Lestrade et al. (1999) and that in the Hipparcos Catalogue, disagrees by $0.80 \pm 0.34$ mas with the revised Hipparcos result of van Leeuwen (2008).

5. The size and phase of the orbit we fit to the stellar radio emission is consistent with that determined for the IM Peg primary from optical spectroscopy by Marsden et al. (2005).

6. Our parameter estimates are sufficiently accurate to ensure that the uncertainty in IM Peg's proper motion makes only a very small contribution to the uncertainty of the $G P-B$ relativity tests.
We thank Bob Campbell for the original version of our WLS fitting software and for his help in making modifications needed for our work. We thank John Chandler, too, for helping with this task and providing the planetary ephemeris used in our parallax calculations. We thank Jingchen Liu and Xiao-Li Meng for Bayesian statistical analyses of our VLBI position estimates. We also thank Norbert Zacharias for discussions and for providing optical astrometric data in the reference frame of the Tycho-2 Catalogue. Finally, we thank the referee for a careful reading of the paper and for valuable comments.

This research was primarily supported by NASA, through a contract from Stanford University to the Smithsonian Astrophysical Observatory (SAO), as well as a major subcontract from SAO to York University, and lesser subcontracts from SAO to the Swiss Federal Institute of Technology Zurich (ETH-Z), Tennessee State University, the University of Pittsburgh, and the U.S. Naval Observatory. The National Radio Astronomy Observatory is a facility of the National Science Foundation operated under cooperative agreement by Associated Universities, Inc. The DSN is operated by JPL/Caltech, under contract with NASA. We have made use of NASA's Astrophysics Data System Abstract Service, initiated, developed, and maintained at SAO.

\section{REFERENCES}

Bartel, N., Bietenholz, M. F., Lebach, D. E., et al. 2012, ApJS, 201, 3 (Paper III) Berdyugina, S. V., Berdyugin, A. V., Ilyin, I., \& Tuominen, I. 2000, A\&A, 360, 272

Berdyugina, S. V., Ilyin, I., \& Tuominen, I. 1999, A\&A, 347, 932

Bietenholz, M. F., Bartel, N., Lebach, D. E., et al. 2012, ApJS, 201, 7 (Paper VII)

ESA. 1997, The HIPPARCOS and TYCHO catalogues, SP-1200 (Noordwijk: ESA)

Everitt, C. W. F., Debra, D. B., Parkinson, B. W., et al. 2011, Phys. Rev. Lett., 106,221101

Fey, A. L., Gordon, D., \& Jacobs, C. S. (ed.) 2009, IERS Technical Note No. 35 (Frankfurt: Verlag des Bundesamts für Kartographie und Geodäsie), http://www.iers.org/IERS/EN/Publications/TechnicalNotes/tn35.html

Franciosini, E., \& Chiuderi Drago, F. 1995, A\&A, 297, 535

Guinan, E. F., \& Giménez, A. 1993, in The Realm of Interacting Binary Stars, ed. J. Sahade, G. E. McCluskey, Jr., \& Y. Kondo (ASSL, Vol. 177; Noordwijk: ESA), 51

Høg, E., Fabricius, C., Makarov, V. V., et al. 2000, A\&A, 355, L27

Lebach, D. E., Ratner, M. I., Shapiro, I. I., et al. 1999, ApJ, 517, L43

Lebach, D. E., Bartel, N., Bietenholz, M. F., et al. 2012, ApJS, 201, 4 (Paper IV)

Lestrade, J.-F., Jones, D. L., Preston, R. A., et al. 1995, A\&A, 304, 182

Lestrade, J.-F., Phillips, R. B., Hodges, M. W., \& Preston, R. A. 1993, ApJ, 410, 808

Lestrade, J.-F., Preston, R. A., Jones, D. L., et al. 1999, A\&A, 344, 1014

Liu, J. 2008, PhD thesis, Harvard University

Marsden, S. C., Berdyugina, S. V., Donati, J.-F., et al. 2005, ApJ, 634, L173

Mayor, M., \& Mazeh, T. 1987, A\&A, 171, 157

Pradel, N., Charlot, P., \& Lestrade, J.-F. 2006, A\&A, 452, 1099

Press, W. H., Teukolsky, S. A., Vetterling, W. T., \& Flannery, B. P. 1992, Numerical Recipes in FORTRAN. The Art of Scientific Computing (2nd ed.; Cambridge: Cambridge Univ. Press)

Ransom, R. R., Bartel, N., Bietenholz, M. F., et al. 2012a, ApJS, 201, 2 (Paper II)

Ransom, R. R., Bartel, N., Bietenholz, M. F., et al. 2012b, ApJS, 201, 6 (Paper VI)

Shapiro, I. I., Bartel, N., Bietenholz, M. F., et al. 2012, ApJS, 201, 1 (Paper I)

Tokovinin, A., Thomas, S., Sterzik, M., \& Udry, S. 2006, A\&A, 450, 681

van Leeuwen, F. 2007, A\&A, 474, 653

van Leeuwen, F. 2008, VizieR Online Data Catalog, 1311, 0 